\title{
Harmonically-Trapped One-Dimensional Fermi Gas Using the Static Fluctuation Approximation
}

\begin{tabular}{|r|l|}
\hline Journal: & Canadian Journal of Physics \\
\hline Manuscript ID & cjp-2015-0315.R1 \\
\hline Manuscript Type: & Article \\
\hline Date Submitted by the Author: & 30 -Aug-2015 \\
\hline Complete List of Authors: & $\begin{array}{l}\text { Al-Khzon, H.A.; Al Imam Mohammad Ibn Saud Islamic University, Physics } \\
\text { Department } \\
\text { Ghassib, H.B.; The University of Jordan, Physics } \\
\text { Al-Sugheir, M.K.; Hashemite University, }\end{array}$ \\
\hline Keyword: & $\begin{array}{l}\text { Harmonically-Trapped systems, Fermi Gas, Static Fluctuation } \\
\text { Approximation, Thermodynamic properties, magnetic properties }\end{array}$ \\
\hline
\end{tabular}

SCHOLARONE ${ }^{m}$

Manuscripts 


\section{Harmonically-Trapped One-Dimensional Fermi Gas Using the Static}

\section{Fluctuation Approximation}

H. A. Al-Khzon ${ }^{\text {a }}$ H. B. Ghassib ${ }^{\text {b }}$ M. K. Al-Sugheir ${ }^{\text {c, d }}$

(a) Physics Department, College of Science, Al Imam Mohammad Ibn Saud Islamic University, Riyadh, Saudi Arabia

(b) Department of Physics, The University of Jordan, Amman, Jordan

(c) Department of Physics, The Hashemite University, Zarqa, Jordan

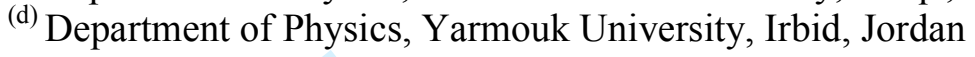

Suggested Running Head: 1D Fermi Gas in SFA

PACS: 65.40.Ba, 67.10.Db, 03.75.Ss, 05.30.Fk, 67.85.Lm

The name and mailing address of the author to whom proofs should be sent:

Prof. Mohamed K. Al-Sugheir

Department of Physics

Faculty of Science

The Hashemite University

Zarqa, Jordan

E-mail:msugh@hu.edu.jo

Tel: (+962) 795609908 


\begin{abstract}
A system of a finite number of harmonically-trapped fermions in one dimension, in the presence of a static magnetic field, is studied within the framework of the static fluctuation approximation, for different repulsive and attractive potential strengths. Specifically, the thermodynamic properties of the system: the chemical potential, total energy, heat capacity and entropy, as well as its magnetic properties: the magnetization and susceptibility, are calculated. It is observed that the system remains in an ordered phase for a small number of particles $N$, even at high temperatures $T$. Disorder sets in for large $N$, even at low $T$. The effect of the potential strength on the heat capacity is particularly tangible in the region bordering the quantum and classical regimes. The effect of the temperature (representing disorder) is directly opposite to that of the magnetic field (representing order), as expected on basic physical grounds. These features are consistent with experimental results.
\end{abstract}




\section{Introduction}

The dimensionality of a system plays a crucial role in determining its physical properties. In low- dimensional systems, the interactions and quantum fluctuations affect the physical properties of the system much more than in higher dimensions: as the particle density decreases, a one-dimensional gas becomes more strongly-interacting - in contrast to the three-dimensional case [1]. So does the finiteness of the system: the total energy, heat capacity, entropy and magnetization are not linearly-dependent on the number of particles - unlike the case for the infinite system, where these quantities are extensive.

The effects of reduced dimensionality have attracted the attention of theorists and experimentalists a like [2-6]. Many theoretical techniques have been applied to onedimensional Fermi systems - including the Bethe ansatz $[7,8]$, the numerically-exact density-matrix renormalization-group approach [9], the spin-density-functional theory [10] and quantum Monte Carlo simulations [11,12]. The one-dimensional two-component Fermi gas in elongated harmonic traps has been the subject of interesting research in the past few years for attractive [13] as well as for repulsive interactions [14]. This system has been studied within the local-density approximation. For attractive interactions, a twoshell structure appears: the Fulde-Ferrell-Larkin-Ovchinnikov state in the center of the trap, and either a fully-paired or a fully-polarized phase in the wings. For repulsive interactions, a partially-polarized phase appears in the center of the trap, and a fullypolarized phase in the wings.

The experimental realization of one-dimensional systems can be achieved by manipulating ultracold quantum gases in atom chips [15-17] and in optical lattices [18, 19]. Different regimes of one-dimensional quantum atomic gases can be explored via the 
well-developed techniques of Feshbach resonances [20, 21]. A recent experimental breakthrough was the realization of a one-dimensional spin-imbalanced Fermi gas of ${ }^{6} \mathrm{Li}$ atoms [22]. Measurements were undertaken for the density profiles of a two-spin mixture of ultracold ${ }^{6} \mathrm{Li}$ atoms trapped in an array of one-dimensional tubes - analogous to electrons in one-dimensional wires. For finite spin imbalance, the system phase-separated with an inverted phase profile, as compared to the three-dimensional case.

Harmonically-trapped atomic gases are inhomogeneous; the number density has a maximum value at the trap center and drops to zero at its edges [23, 24]. For harmonically-trapped Fermi gases, this means that the chemical potential $\mu$ and Fermi temperature $T_{F}$ are position-dependent, although the interatomic collisions should ultimately be sufficient to establish thermal equilibrium.

Experimentally, preparing a homogeneous quantum gas in a trap is still quite a challenging problem. There have been several attempts to achieve this. One class of experiments involves the geometry of the trap; recently it has become possible to produce a homogeneous gas held in the quasi-uniform potential of an optical box trap $[25,26]$. Another class probes a small fraction of atoms near the center of the Fermi cloud; the probed atoms could represent a homogeneous gas [23, 27].

For a 1D homogeneous Fermi gas, at low temperatures, $T<<T_{F}$, all available states in momentum space are filled up to the Fermi 'level' (actually, 'point'), which is sharplydefined. For an inhomogeneous gas, however, the number densities at different positions in a trap give rise to different values of $T_{F}[23]$.

Using the local-density approximation $[27,28]$, the trapped gas can be locally described as a homogeneous gas. That is, each 'local' region in the gas can be considered as a homogeneous system in its own right. A small fraction of atoms near the center of a harmonically-trapped gas could provide a good representation of a homogeneous system 
$[23,29,30]$. It has been found that probing the central $40 \%$ (or less) of the gas is sufficient to approximate a homogeneous Fermi gas $[23,26]$.

In this work, a system of $N$ fermions, each of mass $m$, harmonically-trapped in one dimension, is considered within the static fluctuation approximation (SFA) in the presence of a static magnetic field; $N$ ranges from 50 to 250 . These fermions are assumed to interact through a contact (i.e., delta-function) potential, either attractive or repulsive. SFA has already been applied to several other many-body systems [31-36]. This approach is relatively simple, compared to other many-body theories, in the sense that it is not based on Green's functions or Feynman diagrams. The present formulation of SFA is applicable only to homogeneous systems. Thus, in view of the above remarks, it is valid only for that part of our system located near the center of the Fermi cloud.

Just like SFA, but unlike the Gross-Pitaevskii equation [37], the Bogoliubov-de Gennes (BdG) approximation [1] takes into account the quantum fluctuations which cannot be ignored, specifically in low-dimensional systems. The BdG approximation is particularly suitable for inhomogeneous Fermi systems [38].

The paper is organized as follows. In Section 2, the rudiments of SFA are outlined. In Section 3 , the thermodynamic properties comprising the chemical potential $\mu$, total energy $U$, heat capacity $C$ and entropy $S$, as well as the magnetic properties including the magnetization $M$ and susceptibility $\chi$, are presented and discussed. Some closing remarks follow in Section 4.

\section{SFA for a One-dimensional Trapped Fermi Gas}

\subsection{The Hamiltonian}

The total Hamiltonian of the one-dimensional trapped Fermi gas is given by

$$
\hat{H}=\hat{H}_{0}+\hat{H}_{1} .
$$


$\hat{H}_{0}$ is the single-particle Hamiltonian and $\hat{H}_{1}$ is the interaction term:

$$
\begin{aligned}
& \hat{H}_{0}=\int \hat{\Psi}^{+}(x)\left[\frac{-\hbar^{2}}{2 m} \frac{\partial^{2}}{\partial x^{2}}+\frac{1}{2} m \omega_{x}^{2} x^{2}\right] \hat{\Psi}(x) d x ; \\
& \hat{H}_{1}=\frac{1}{2} \iint \hat{\Psi}^{+}\left(x_{1}\right) \hat{\Psi}^{+}\left(x_{2}\right) V\left(x_{1}-x_{2}\right) \hat{\Psi}\left(x_{2}\right) \hat{\Psi}\left(x_{1}\right) d x_{1} d x_{2} .
\end{aligned}
$$

$\hat{\Psi}(x)$ and $\hat{\Psi}^{+}(x)$ are the Fermi field operators and $\omega_{x}$ is the frequency in the longitudinal direction:

$$
\hat{\Psi}(x)=\sum_{k, \lambda} \phi_{k}(x) \eta_{\lambda} \hat{b}_{k \lambda} ; \quad \hat{\Psi}^{+}(x)=\sum_{k, \lambda} \phi_{k}^{*}(x) \eta_{\lambda} \hat{b}_{k \lambda}^{+},
$$

where the coefficients $\phi_{k}(x)$ are the single-particle wave functions and $\eta_{\lambda}$ is the spin wave function; $\hat{b}_{k \lambda}^{+}$and $\hat{b}_{k \lambda}$ are the creation and annihilation operators, respectively. The sum is over the complete set of single-particle quantum numbers, including spin. For a harmonically-trapped one-dimensional system:

$$
\phi_{k}(x, \alpha)=\frac{\alpha^{1 / 4}}{\pi^{\frac{1}{4}} \sqrt{2^{k} k !}} \exp \left(\frac{-\alpha}{2} x^{2}\right) H_{k}(\sqrt{\alpha} x) .
$$

$\alpha=m \omega_{x} / \hbar$; the coordinates are measured in units of the oscillator length; and $H_{k}(x)$ is the Hermite polynomial of order $k$ and argument $x$.

For mathematical convenience, the interatomic interaction is taken to be the contact potential:

$$
V\left(x_{1}-x_{2}\right)=g \delta\left(x_{1}-x_{2}\right),
$$

$g$ being the potential strength determined from the $s$-wave scattering length.

In the presence of an external magnetic field $B$ (assumed here to be static), another term should be added to $\hat{H}$ so as to take into account the spin-field interaction:

$$
\hat{H}_{2}= \pm \mu_{B} B
$$

$\mu_{B}$ being the Bohr magneton, the positive (negative) sign corresponding to antiparallel (parallel) magnetic moment to the field. Thus, 


$$
\hat{H}=\hat{H}_{0}+\hat{H}_{1}+\hat{H}_{2}=\sum_{k \lambda}^{\infty} \varepsilon(k \lambda) \hat{b}_{k \lambda}^{+} \hat{b}_{k \lambda}+\frac{g}{2} \sum_{\substack{m_{1}, m_{2}, m_{3} \\ m_{4}, \lambda_{1}, \lambda_{2}}}^{\infty} C\left(m_{1}, m_{2}, m_{3}, m_{4}\right) \hat{b}_{m_{1}, \lambda_{1}}^{+} \hat{b}_{m_{2}, \lambda_{2}}^{+} \hat{b}_{m_{3}, \lambda_{2}} \hat{b}_{m_{4}, \lambda_{1}},
$$

where the single-particle energy, incorporating $\mu$, is

$$
\varepsilon(k \lambda)=\hbar \omega\left(k+\frac{1}{2}\right)-\mu \pm \mu_{B} B
$$

and the matrix elements $C\left(m_{1}, m_{2}, m_{3}, m_{4}\right)$ are given by

$$
C\left(m_{1}, m_{2}, m_{3}, m_{4}\right)=\int \phi_{m_{1}}(x) \phi_{m_{2}}(x) \phi_{m_{3}}(x) \phi_{m_{4}}(x) d x
$$

\subsection{SFA Formalism}

In the well-known mean-field approximation, the local-field operator is replaced with its mean value; whereas, in SFA, it is the square of this operator that is replaced with its mean value. The physical implication is that the corresponding energy spectrum in the latter case is replaced with a distribution around the mean value [31-36, 39-45].

SFA has been applied to both strongly- and weakly-interacting systems [31-36, 39-45]. The formalism can, in principle, be applied to any many-body system. However, it is ultimately an independent particle model; it can be viewed as a modified mean-field approach. Thus, its validity is dubious for dense and strongly-interacting systems - except perhaps at sufficiently low $T$ [44]. The local-field (energy) operator can be written in terms of its mean value and the corresponding fluctuations: $\hat{E}_{k \lambda}=\left\langle\hat{E}_{k \lambda}\right\rangle+\Delta \hat{E}_{k \lambda}$. The basic assumption in SFA is that, as has just been mentioned, the square of the fluctuations in this operator is replaced with its mean value: $\Delta \hat{E}_{k \lambda}^{2}=\left\langle\Delta \hat{E}_{k \lambda}^{2}\right\rangle=\Omega_{k \lambda}^{2}$. In the mean-field approximation, it is the operator itself that is replaced with its mean value $\left\langle\hat{E}_{k \lambda}\right\rangle$; SFA, then, goes one step further. Its validity depends on how much fluctuation there is relative to the mean value of the operator. These fluctuations depend on both $T$ and $g$. At low $T$ or for weakly-interacting systems, i.e., for small magnitudes of $g$, the results obtained within 
SFA are expected to be credible.

In SFA, the Hamiltonian $\hat{H}$ can be written as a linear combination of the hermitian operator $\hat{E}_{k \lambda}(\tau)$ and the occupation number operator $\hat{n}_{k \lambda}=\hat{b}_{k \lambda}^{+} \hat{b}_{k \lambda}$ :

$$
\hat{H}=\sum_{k \lambda} \hat{E}_{k \lambda}(\tau) \hat{b}_{k \lambda}^{+}(\tau) \hat{b}_{k \lambda}(\tau)
$$

Here $\tau \equiv i t, t$ being the time, and the indices $k \lambda$ denote a complete set of quantum numbers describing a specific state.

Another basic assumption of SFA is that the local-field operator commutes with $\hat{b}_{k \lambda}^{+}$and $\hat{b}_{k \lambda}$. It follows that the equation of motion for $\hat{b}_{k \lambda}^{+}(\tau)$ in the Heisenberg picture is, using Eq. (11),

$$
\frac{d \hat{b}_{k \lambda}^{+}(\tau)}{d \tau}=\left[\hat{H}, \hat{b}_{k \lambda}^{+}(\tau)\right]=\hat{E}_{k \lambda}(\tau) \hat{b}_{k \lambda}^{+}(\tau)
$$

From Eqs. (8) and (12), $\hat{E}_{k \lambda}(\tau)$ is found to be [32]

$$
\left.\hat{E}_{\mathrm{k} \lambda}=\left\{\hat{b}_{k \lambda}, \mid \hat{H}, \hat{b}_{k \lambda}^{+}\right]\right\}=\varepsilon(k \lambda)+g \sum_{\substack{m_{1}=0 \\ \lambda^{\prime} \neq \lambda}} C\left(k, m_{1}\right) \hat{b}_{m_{1} \lambda}^{+} \hat{b}_{m_{1} \lambda^{\prime}}
$$

where the matrix element $C\left(k, m_{1}\right)$ is

$$
C\left(k, m_{1}\right)=C\left(k, m_{1}, k, m_{1}\right)=\int_{-\infty}^{\infty} \phi_{k}^{2}(x) \phi_{m_{1}}^{2}(x) d x
$$

One can then obtain all the necessary equilibrium correlation functions from the so-called long-range equation [44], which relates the mean value of $\hat{n}_{k \lambda}$ to that of $\hat{E}_{k \lambda}$ :

$$
\left\langle\hat{n}_{k \lambda} \hat{A}\right\rangle=\eta_{0}(k \lambda)\langle\hat{A}\rangle+\eta_{1}(k \lambda)\left\langle\Delta \hat{E}_{k \lambda} \hat{A}\right\rangle
$$

$\hat{A}$ being an arbitrary operator which commutes with $\hat{b}_{k \lambda}^{+}$and $\hat{b}_{k \lambda} ; \sqrt{\left\langle\left(\Delta \hat{E}_{k \lambda}(\tau)\right)^{2}\right\rangle}$ has only the characteristic values $\pm \Omega_{k \lambda}$ [cf. Eq. (20) below];

$$
\eta_{0}(k \lambda) \equiv \frac{1}{2}\left[\frac{1}{\exp \left[\beta\left(\left\langle\hat{E}_{k \lambda}\right\rangle+\Omega_{k \lambda}\right)\right]+1}+\frac{1}{\exp \left[\beta\left(\left\langle\hat{E}_{k \lambda}\right\rangle-\Omega_{k \lambda}\right)\right]+1}\right]
$$




$$
\eta_{1}(k \lambda) \equiv \frac{1}{2 \Omega_{k \lambda}}\left[\frac{1}{\exp \left[\beta\left(\left\langle\hat{E}_{k \lambda}\right\rangle+\Omega_{k \lambda}\right)\right]+1}-\frac{1}{\exp \left[\beta\left(\left\langle\hat{E}_{k \lambda}\right\rangle-\Omega_{k \lambda}\right)\right]+1}\right] .
$$

where $\beta=1 / k_{B} T, k_{B}$ being Boltzmann's constant.

The distribution function can be determined by putting $\hat{A}=1$ in Eq. (15):

$$
\left\langle\hat{n}_{k \lambda}\right\rangle=\eta_{0}(k \lambda)
$$

It is more convenient to rewrite the 'generating' equation (15) in terms of the deviation of the occupation number operator, defined as

$$
\Delta \hat{n}_{k \lambda}=\left\langle\hat{n}_{k \lambda}\right\rangle-\hat{n}_{k \lambda} .
$$

Thus,

$$
\left\langle\Delta \hat{n}_{k \lambda} \hat{A}\right\rangle=-\eta_{1}(k \lambda)\left\langle\Delta \hat{E}_{k \lambda} \hat{A}\right\rangle \text {. }
$$

The square of the fluctuation in the local-field operator can be calculated from Eq. (13):

$$
\Omega_{k \lambda}^{2}=\left\langle\left(\Delta \hat{E}_{k \lambda}\right)^{2}\right\rangle=g^{2} \sum_{\substack{m_{1}, m_{2} \\ \lambda^{\prime} \neq \lambda}} C\left(k, m_{1}\right) C\left(k, m_{2}\right)\left\langle\Delta \hat{n}_{m_{1} \lambda^{\prime}} \Delta \hat{n}_{m_{2} \lambda^{\prime}}\right)
$$

for spin-up particles:

$$
\left.\Omega_{k_{\uparrow} \uparrow}^{2}=g^{2} \sum_{m_{1}=0} C^{2}\left(k, m_{1}\right)\left\langle\left(\Delta \hat{n}_{m_{1} \downarrow}\right)^{2}\right\rangle+g^{2} \sum_{m_{1}, m_{2}} C\left(k, m_{1}\right) C\left(k, m_{2}\right)\left\langle\Delta \hat{n}_{m_{1} \downarrow} \downarrow \Delta \hat{n}_{m_{2} \downarrow}\right\rangle\right)_{c}
$$

and for spin-down particles:

$$
\Omega_{k \downarrow}^{2}=g^{2} \sum_{m_{1}=0} C^{2}\left(k, m_{1}\right)\left\langle\left(\Delta \hat{n}_{m_{1} \uparrow}\right)^{2}\right\rangle+g^{2} \sum_{m_{1}, m_{2}} C\left(k, m_{1}\right) C\left(k, m_{2}\right)\left\langle\Delta \hat{n}_{m_{1} \uparrow}\left\langle\hat{n}_{m_{2} \uparrow}\right\rangle_{c}\right.
$$

Further, the square of the fluctuation in the number of particles is

$$
\left\langle\left(\Delta \hat{n}_{k \lambda}\right)^{2}\right\rangle=\left\langle\left(\hat{n}_{k \lambda}\right)^{2}\right\rangle-\left\langle\hat{n}_{k \lambda}\right\rangle^{2}
$$

For Fermi systems, $\hat{n}_{k \lambda}^{2}=\hat{n}_{k \lambda}$; so that Eq.(23) becomes

$$
\left\langle\left(\Delta \hat{n}_{k \lambda}\right)^{2}\right\rangle=\left\langle\hat{n}_{k \lambda}\right\rangle\left[1-\left\langle\hat{n}_{k \lambda}\right\rangle\right]
$$

To find the pair correlation function between spin-up fermions, we put $\hat{A}=\Delta \hat{n}_{k \lambda}$ in Eq. (19): 


$$
\left\langle\Delta \hat{n}_{m_{1} \uparrow} \Delta \hat{n}_{m_{2} \uparrow}\right\rangle_{c}=-g \eta_{1}\left(m_{1}, \uparrow\right) \sum_{i} C\left(m_{1}, i\right)\left\langle\Delta \hat{n}_{i \downarrow} \Delta \hat{n}_{m_{2} \uparrow}\right\rangle
$$

similarly for spin-down particles:

$$
\left\langle\Delta \hat{n}_{m_{1} \downarrow} \Delta \hat{n}_{m_{2} \downarrow}\right\rangle_{c}=-g \eta_{1}\left(m_{1}, \downarrow\right) \sum_{i} C\left(m_{1}, i\right)\left\langle\Delta \hat{n}_{i \uparrow} \Delta \hat{n}_{m_{2} \downarrow}\right\rangle ;
$$

and for the correlation function between spin-down and spin-up fermions:

$$
\left\langle\Delta \hat{n}_{i \downarrow} \Delta \hat{n}_{m_{2} \uparrow}\right\rangle=-g \eta_{1}(i, \downarrow) C\left(i, m_{2}\right)\left\langle\left(\Delta \hat{n}_{m_{2} \uparrow}\right)^{2}\right\rangle-g \eta_{1}(i, \downarrow) \sum_{f} C(i, f)\left\langle\Delta \hat{n}_{f \uparrow} \Delta \hat{n}_{m_{2} \uparrow}\right\rangle_{c}
$$

From the closed set of nonlinear coupled equations (17,19-27), one can find $\left\langle\hat{E}_{k \lambda}\right\rangle,\left\langle\hat{n}_{k \lambda}\right\rangle,\left\langle\left(\Delta \hat{n}_{k \lambda}\right)^{2}\right\rangle,\left\langle\Delta \hat{n}_{k \lambda}, \Delta \hat{n}_{q \lambda^{\prime}}\right\rangle_{c}$ and $\Omega_{k \lambda}$. The resulting coupled equations were solved numerically, using an iterative procedure. Throughout the work, trap units were used: $E=\frac{E}{\hbar \omega}$ and $g_{e f f}=\frac{g}{\hbar \omega}$.

A number of states $m$ was used up to 2000, as discussed in [45], such that the results were independent of $m$. Further, Eq. (6) could not be used directly to evaluate the wave functions $\phi_{m}(x)$ because of the extreme numerical difficulty in calculating $m$ ! for relatively high orders. This problem was solved with the aid of the following recursion relation (which can be derived at once from the analogous relation for Hermite polynomials):

$\phi_{m+1}(x)=\sqrt{\frac{2}{m+1}} x \phi_{m}(x)-\sqrt{\frac{m}{m+1}} \phi_{m-1}(x)$.

To calculate the thermodynamic properties of the system, we start with the grand partition function $Q$, defined as

$$
\begin{aligned}
Q & =\operatorname{Tr}(\exp (-\beta \hat{H})) . \\
& =\sum_{\hat{n}_{p}} \exp \left(-\beta \sum_{p \lambda} \hat{E}_{p \lambda} \hat{n}_{p \lambda}\right) \\
& =\prod_{p \lambda} \sum_{n_{p \lambda}} \exp \left(-\beta \hat{E}_{p \lambda} \hat{n}_{p \lambda}\right)
\end{aligned}
$$

For Fermi systems $n_{p \lambda}=0$ or 1 ; so that (29) becomes 


$$
Q=\prod_{p \lambda}\left(1+\exp \left(-\beta \hat{E}_{p \lambda}\right)\right)
$$

It is more convenient to take the logarithms of both sides:

$$
\ln Q=\sum_{p \lambda} \ln \left(1+\exp \left(-\beta \hat{E}_{p \lambda}\right)\right) \text {. }
$$

This equation can be rewritten as linear in terms of the fluctuations of the local-field operator [44]; with the aid of the identity

$$
F\left(a+b \Delta \hat{E}_{p \lambda}\right)=q_{0}(p, \lambda)+q_{1}(p, \lambda) \Delta \hat{E}_{p \lambda},
$$

we have

$$
\ln Q=\sum_{p \lambda}\left(q_{0}(p, \lambda)+q_{1}(p, \lambda) \Delta \hat{E}_{p \lambda}\right) .
$$

Taking into account the symmetry of the quadratic fluctuations of the field operator, we get

$$
\ln Q=\sum_{p \lambda} q_{0}(p, \lambda)
$$

where

$$
q_{0}(p, \lambda)=\frac{1}{2}\left[\ln \left(1+\exp \left(-\beta\left(\left\langle\hat{E}_{p \lambda}\right\rangle+\Omega \text { p }\right)\right)\right)+\ln \left(1+\exp \left(-\beta\left(\left\langle\hat{E}_{p \lambda}\right\rangle-\Omega_{p \lambda}\right)\right)\right)\right] .
$$

The grand mean energy $E$ is given by

$$
E=\langle\hat{H}\rangle=-\frac{\partial}{\partial \beta} \ln Q=\sum_{p \lambda}\left(\left\langle\hat{E}_{p \lambda}\right\rangle\left\langle\hat{n}_{p \lambda}\right\rangle+\eta_{1}(p, \lambda) \Omega_{p \lambda}^{2}\right)=U-\mu N,
$$

$U$ being the total energy and $N$ the total number of particles.

The Helmholtz free energy $A$ of the system is defined as

$$
A=N \mu-k_{B} T \ln Q
$$

and the entropy $S$ as

$$
S=\frac{U-A}{T}
$$

The heat capacity at constant volume is

$$
C=\left(\frac{\partial U}{\partial T}\right)_{V} \text {. }
$$

Finally, the magnetization $\mathrm{M}$ of the system is given by

$$
\frac{M}{N \mu_{B}}=\frac{N_{\uparrow}-N_{\downarrow}}{N}
$$

$N_{\uparrow}$ and $N_{\downarrow}$ being the numbers of up-spins and down-spins, respectively.

3. Results and Discussion

\subsection{Thermodynamic Properties}

\subsubsection{Chemical potential $\mu$}

This was calculated from the constraint that the total number of particles in the system is 
constant:

$$
N=\sum_{k, \lambda}\left\langle\hat{n}_{k, \lambda}\right\rangle=\frac{1}{2}\left\{\frac{1}{\left.\exp \beta \beta\left(\left|\hat{E}_{k \lambda}\right\rangle+\Omega_{k \lambda}\right)\right]+1}+\frac{1}{\exp \left\{\left(\left|\hat{E}_{k \lambda}\right\rangle-\Omega_{k \lambda}\right)\right]+1}\right\} .
$$

Figure 1 shows $\mu$, scaled by the Fermi energy of the noninteracting system $E_{F}$, as a function of $T / T_{F}$ at frequency $\omega=200 \mathrm{~Hz}$, magnetic field $B=2 n T$ and potential strength $g_{\text {eff }}=0.1$ for different $N, T_{F}$ being the Fermi temperature of the trapped noninteracting system: $\mathrm{T}_{\mathrm{F}}=\frac{E_{F}}{k_{B}} ; E_{F}=\frac{N \hbar \omega}{2}[46]$. The general behavior of $\frac{\mu}{E_{F}}$ with $T / T_{F}$ is the same, regardless of $N$. At low $T, \mu$ is almost constant and equal to $E_{F}$. As $T$ increases, fermions in energy states close to $E_{F}$ are excited to states just above the Fermi level, leaving holes just below this level. Thus, as $T$ increases, $\mu$ decreases. At high $T, \mu$ goes to its classical value and becomes negative. The behavior of $\mu / E_{F}$ with $T / T_{F}$ indicates that $\mu$ increases as $N$ increases; $T_{F}$ is linearly dependent on $N$. This agrees with previous results obtained using Feynman-diagrammatic techniques [47].

The effect of different repulsive and attractive $g_{\text {eff }}$ on $\mu / E_{F}$ at $\omega=200 \mathrm{~Hz}, B=2 n T$ and $N=$ 150 is displayed in Fig. 2. It is observed that the general behavior of $\mu$ is the same, regardless of $g_{\text {eff. }}$ For weak $g_{\text {eff, }} \mu$ is quite close to its value for the noninteracting harmonically-trapped Fermi gas. As $g_{\text {eff }}$ grows stronger, $\mu$ shifts up (down) for repulsive (attractive) interactions. This result is expected since the energy spectrum of the system is linearly-dependent on $g_{\text {eff, }}$, and $E_{F}$ shifts up (down) for repulsive (attractive) interactions. Su et al. [48] studied the low- $T$ behavior of a weakly-interacting Fermi gas trapped in a power-law potential, based on the pseudopotential method and the local-density approximation. For the interacting Fermi system trapped in a harmonic potential, they found that the repulsive (attractive) interactions increased (decreased) $\mu$, just as in our work.

The results for $\mu / E_{F}$ as a function of $T / T_{F}$ at $N=150, B=2 n T$ and $g_{\text {eff }}=0.1$ for different 
$\omega$ are presented in Fig. 3. Regardless of $\omega, \mu / E_{F}$ versus $T / T_{F}$ exhibits the same behavior. This means that $\mu$ is linearly-dependent on $\omega$, just as in a trapped noninteracting system. For the present values of $g_{\text {eff, }}$ the trapping energy dominates, as compared to the interaction energy.

Figure 4 shows $\mu / E_{F}$ as a function of $B$ at $g_{\text {eff }}=0.1, T=22.6 n K$ and $\omega=200 \mathrm{~Hz}$ for different $N$. At low $B, \mu / E_{F}$ is constant. The energy spectrum of the system, Eq. (13), is almost linearly-dependent on $B$. At low $B, \mu_{B} B<\hbar \omega$, the effect of the magnetic term on $\mu$ will be small. As $B$ increases, this effect increases, with a corresponding increase in the number of particles having magnetic moments parallel to $B$. It follows that more excited states become occupied; so that $\mu / E_{F}$ decreases linearly as $B$ increases. At high $B$, most particles will have magnetic moments parallel to $B$; the magnetic term, $-\mu_{B} B$, in the energy spectrum then dominates, and $\mu$ becomes negative.

The thermodynamic properties of a noninteracting, harmonically-trapped Fermi gas were calculated by Grether et al. [49]. They found that at zero $T, \mu$ was equal to $E_{F}$, as expected; it then decreased with increasing $T$, becoming negative at $T_{F}$. The consistency in behavior between these results and ours may indicate that the hard-core interaction does not affect this behavior.

In concluding this subsection, we note that by examining the behavior of $\mu$, we are in effect studying the fundamental thermodynamic function, namely, the Gibbs free energy $G$, since $G=N \mu$.

\subsubsection{Total energy $U$}

Figure 5 shows the total energy per particle, $U / N$, in trap units as a function of $T / T_{F}$ at $\omega=200 H z, B=2 n T$ and $g_{\text {eff }}=0.1$ for different $N$.

At low $T$, most particles accumulate in energy states lower than the Fermi energy (i.e., in 
the ground state or the degenerate regime); $U / N$ will then be almost constant. As $T$ increases, the number of particles in excited states increases; so that $U / N$ increases. $U / N$ also increases as $N$ increases. This increase becomes more pronounced as $T$ increases even further. For finite systems, $U / N$ is an $N$-dependent quantity; unlike the case for infinite systems, where $U / N$ is intensive. In a previous study [50], $U / N$ was investigated for a polarized atomic Fermi gas in a highly-elongated harmonic trap, using a mean-field Bogoliubov-de Gennes theory; the results are consistent with ours.

Figure 6 shows $U / N$ in trap units as a function of $T / T_{F}$ at $\omega=200 H z, B=2 n T$ and $N=$ 150 for different repulsive and attractive $g_{\text {eff. }}$ Regardless of $g_{\text {eff, }}$ the general behavior of $U / N$ has the same profile. This indicates that repulsive (attractive) potentials shift up (down) the energy spectrum of the system. At very low $T$ and weak $g_{\text {eff }}$, most particles are in the ground state; so that $U / N$ is almost constant. For attractive (repulsive) potentials, as $g_{\text {eff }}$ increases, the interaction term will reduce (increase) the value of $U / N$. At high $T$, the values of $U / N$ are close to each other for all $g_{\text {eff, }}$ since the thermal energy dominates. The energy per particle for the interacting, harmonically-trapped Fermi gas was computed as a function of $T$ using three approaches: the mean-field approximation [51], the Bogoliubovde Gennes approximation [1], and the local-density approximation [48]. In addition, this quantity was calculated for the noninteracting system [49]. The results were similar in all these four cases. The energy per particle was almost constant at low $T$ and increased monotonically with increasing $T$, becoming linear in the classical limit. The agreement between these results and ours may imply that the interaction does not change the behavior of the thermodynamic properties; it just shifts them.

Figure 7 shows $U / N$ in trap units as a function of $T / T_{F}$ at $N=150, B=2 n T$ and $g_{\text {eff }}=0.1$ for different $\omega$. The energy per particle in trap units as a function $T / T_{F}$ is independent of $\omega$. At low $T, U / N$ is almost constant; but goes linear with $T$ at high $T$, just like the classical system. 
Figure 8 displays $U / N$ in trap units as a function of $B$ at $g_{\text {eff }}=0.1, T=22.6 n K$ and $\omega=$ $200 \mathrm{~Hz}$ for different $N$. At low $B, U / N$ is almost constant; $B$ is insufficient to excite particles above the Fermi level. As $B$ increases, $U / N$ decreases linearly; this is because more particles will have magnetic moments parallel to the field, and the energy spectrum shifts down. At very large $B, \mu_{B} B>\hbar \omega$, most particles will have their magnetic moments parallel to the field; so that the magnetic energy term in the energy spectrum will dominate. Thus, in this case, $U / N$ becomes negative.

\subsubsection{Heat capacity $C$}

Figure 9 shows the heat capacity $C / N k_{B}$ as a function of $T / T_{F}$ at $\omega=200 \mathrm{~Hz}, B=2 n T$ and $g_{\text {eff }}=0.1$ for different $N$. The general behavior of $C$ has the same profile. It increases monotonically until it reaches a plateau at $T \sim T_{F}$. Butts and Rokhsar [52] and Li et al. [53] studied the properties of a spin-polarized Fermi gas in a harmonic trap, using the ThomasFermi approximation. They found that $C$ was a monotonic function of $T$, and in the high- $T$ limit $C=3 N k_{B}$. According to our SFA calculations, $C$ for the present (one-dimensional) system is also a monotonic function at low $T$ and reaches the classical value $C=N k_{B}$ at high $T$, just as in the noninteracting system [49].

Figure 10 shows $C / N k_{B}$ as a function of $T / T_{F}$ at $N=150, \omega=200 H z$ and $B=2 n T$ for different repulsive and $g_{\text {eff. }}$ The general behavior of $C$ with $T$ is the same, regardless of $g_{\text {eff. }}$ The effect of $g_{\text {eff }}$ on $C$ is tangible in the region bordering the quantum and classical regimes. For attractive potentials, the system's transition to the classical regime is faster than for repulsive potentials because $E_{F}$ shifts up (down) for repulsive (attractive) potentials. Similar results were found by Su et al. [48].

In passing, it is worth mentioning that $C$ was calculated as a function of $T$ for a $d$ dimensional Fermi gas trapped by $\delta(<d)$ mutually-perpendicular harmonic potentials 
[49]. It was found that as $\delta$ increased, $C$ increased.

Figure 11 shows $C / N k_{B}$ as a function of $T / T_{F}$ at $N=150, B=2 n T$ and $g_{\text {eff }}=0.1$ for different $\omega$. As $\omega$ increases, the transition temperature from the quantum to the classical regime increases; $T_{F}$ increases with $\omega$.

Figure 12 displays $C / N k_{B}$ as a function of $T / T_{f}$ at $\omega=200 H z, N=150$ and $g_{\text {eff }}=0.1$ for different $B$. $C$ has almost the same profile, regardless of $B$. It reaches its classical value at $T_{F}$. A slight difference is observed between the results below $0.5 T_{F}$ and those above it. For low $T, T<0.5 T_{F}, C$ decreases with increasing $B$; whereas for high $T . T>0.5 T_{F}, C$ increases with increasing $B$. This is because the energy spectrum of the system consists of three terms: the trapping, magnetic and interaction terms; and so does the heat capacity. Now, $B$ affects both the energy spectrum and the particle distribution. For $T<0.5 T_{F}$, the total energy per particle increases slowly as $B$ increases because the trapping energy dominates; whereas for $T>0.5 T_{F}$, the total energy per particle increases faster with $T$ as $B$ increases because the thermal energy is dominant.

\subsubsection{Entropy $S$}

Figure 13 shows the entropy $S / N k_{B}$ as a function of $T / T_{F}$ at $\omega=200 \mathrm{~Hz}, B=2 n T$ and $g_{\text {eff }}=$ 0.1 for different $N$. At low $T, S$ is affected by neither $T$ nor $N$; this is why we calculated $S$ up to $30 T_{F}$. As $T \rightarrow 0, S / N k_{B} \rightarrow 0$ for all values of $N$, in accordance with the third law of thermodynamics. With $T$ increasing, $S / N k_{B}$ increases. Our entropy results are consistent with those of the Bethe ansatz [54].

Figure 14 shows $S / N k_{B}$ as a function of $T / T_{F}$ at $\omega=200 H z, B=2 n T$ and $N=150$ for different repulsive and attractive $g_{\text {eff. }} S / N k_{B}$ has the same behavior, regardless of $g_{\text {eff. }}$ As $T \rightarrow 0, S / N k_{B} \rightarrow 0$. With $T$ increasing, $S / N k_{B}$ increases. The interaction term in the energy spectrum slightly shifts up (down) $E_{F}$ for repulsive (attractive) potentials. 
Figure 15 shows $S / N k_{B}$ as a function of $T / T_{F}$ at $N=150, B=2 n T$ and $g_{\text {eff }}=0.1$ for different $\omega$. The results overlap exactly; $S / N k_{B}$ is independent of $\omega$.

Figure 16 shows $S / N k_{B}$ as a function of $B$ at $g_{\text {eff }}=0.1, T=2.14 \mu \mathrm{K}$ and $\omega=200 \mathrm{~Hz}$ for different $N$. At low $B, S / N k_{B}$ has a maximum. As $B$ increases, $S / N k_{B}$ decreases since more magnetic dipole moments in this case align themselves along the field.

We close this subsection by stating that the above results also apply to the fundamental thermodynamic functions, the Helmholtz free energy $A=U-T S$, and the enthalpy $H=$ $N \mu+T S$.

\subsection{Magnetic Properties}

\subsubsection{Magnetization $M$}

Figure 17 shows the magnetization $M / N \mu_{B}$ as a function of $T / T_{f}$ at $\omega=200 \mathrm{~Hz}, B=2 n T$ and $g_{\text {eff }}=0.1$ for different $N$. At $T \rightarrow 0, M / N \mu_{B}$ has a maximum. As $T$ increases, $M / N \mu_{B}$ decreases gradually, going to zero at high $T$. The explanation lies, as usual with such phenomena, in the competition between $B$ (as a measure of order) and $T$ (as a measure of disorder). The increase in $M / N \mu_{B}$ with decreasing $N$ is consistent with our results for $S$, the system becoming more ordered when $N$ decreases.

Figure 18 shows $M / N \mu_{B}$ as a function of $T / T_{F}$ at $\omega=200 H z, B=2 n T$ and $N=150$ for different repulsive and attractive $g_{\text {eff. }}$ At low $T\left(<0.25 T_{F}\right)$, three cases suggest themselves. The first is when $g_{\text {eff }}$ is small: the system is almost ideal, and the magnetization is approximately constant. The second case is for attractive potentials: as $g_{\text {eff }}$ increases, $M$ decreases from its 'ideal value', since attractive interactions hamper the alignment of magnetic moments along the field; however, as $T$ increases, the thermal energy balances the effect of the attractive potential, so that $M$ increases to reach a maximum. The third case is for repulsive potentials; as $g_{\text {eff }}$ increases, $M$ decreases rapidly with increasing $T$. At 
high $T\left(>0.25 T_{F}\right)$, the thermal energy counteracts the effect of the potential, and the values of $M$ are close to each other regardless of the potential strength and type, going to zero at very high $T$.

Figure 19 displays $M / N \mu_{B}$ as a function of $T / T_{F}$ at $N=150, B=2 n T$ and $g_{\text {eff }}=0.1$ for different $\omega$. Now, $E_{F}$ is linearly-dependent on $\omega$. The magnetic term in the energy spectrum plays a crucial role at low $\omega$ (weakly-trapped system): the field is able to order the magnetic moments at low $\omega$, especially at low $T$. This explains the results in Fig. 20. At high $T$, the thermal energy disrupts the order of the system.

Figure 20 displays $M / N \mu_{B}$ as a function of $B$ at $g_{\text {eff }}=0.1, T=22.6 n K$ and $\omega=200 \mathrm{~Hz}$ for different $N . M / N \mu_{B}$ increases with increasing $B$ until it reaches a saturation value when all the magnetic moments are oriented parallel to the field.

\subsubsection{Magnetic susceptibility $\chi$}

Figure 21 shows the susceptibility $\chi / N \mu_{B}$ as a function of $T / T_{F}$ at $\omega=200 \mathrm{~Hz}, B=2 n T$ and $g_{\text {eff }}=0.1$ for different $N$. At low $T$ and small $N, \chi / N \mu_{B}$ saturates at finite values. This can be understood from the behavior of the Fermi distribution function: only particles that have energies around $E_{F}$ can change their spin orientation in response to the external field. As $T$ increases, $\chi / N \mu_{B}$ decreases from its maximum value until it reaches zero at high $T$, as before. The curves in this figure are 'proportional' to the corresponding curves in Fig. 17, indicating that the system under the present physical conditions is paramagnetic. This is consistent with experiment [24].

\section{Conclusion}

The thermodynamic and magnetic properties of a one-dimensional harmonically-trapped Fermi system in the presence of a static magnetic field were calculated within the 
framework of the static fluctuation approximation as functions of the temperature and magnetic field for different physical characteristics of the system. The set of nonlinear coupled equations involved was solved numerically by an iteration method for different repulsive and attractive potential strengths. Our main conclusions are: (1) the effect of the potential strength on the heat capacity is particularly tangible in the region bordering the quantum and classical regimes; (2) the effect of the temperature (representing disorder) is directly opposite to that of the magnetic field (representing order), as expected from basic physical considerations; (3) the present system is paramagnetic.

In their overall behavior, our SFA results conform with those of Liu et al. [50]. This latter work is based on the BdG approximation for a polarized atomic Fermi gas in a highlyelongated harmonic trap. Our thermodynamic results agree in general with those of the quantum Monte Carlo method [12]. The calculation of the magnetic properties of the system in the present work represents a bonus.

The behavior of our thermodynamic properties as a function of $T$ for weak $g_{\text {eff }}$ is close to that of a trapped ideal Fermi gas at ultra-low finite temperature studied by Qin and Chen [55], using the fractional exclusion statistics of Haldane and $\mathrm{Wu}$.

\section{Acknowledgment}

M. K. Al-Sugheir is grateful to The Hashemite University for granting him a sabbatical leave in the academic year 2014-2015, during which this work was completed. 


\section{References}

[1] H. Moritz, (2006) Unpublished Doctoral Dissertation. University of Heidelberg, Germany.

[2] M. Gaudin, Phys. Lett. A 24 (1967) 55-56.

[3] C. N. Yang, Phys. Rev. Lett. 19 (1967) 1312-1315.

[4] X. W. Guan, M. T. Batchelor, C. Lee, M. Bortz, Phys. Rev. B 76 (2007) 08512.

[5] H. Hu, Xia-Ji. Liu, P. D. Drummond, Phys. Rev. Lett. 98 (2007) 070403.

[6] K. Gunter, Th. Stoferle, H. Moritz, M. Kohl, and T. Esslinger, Phys. Rev. Lett. 95 (2005) 230401.

[7] G. Orso, Phys. Rev. Lett. 98 (2007) 070403.

[8] P. He, X. Yin, X. Guan, T. M. Batchelor, Y. Wang, Phys. Rev. A 82 (2010) 053633.

[9] M. Tezuka, M. Ueda, New. J. Phys. 12 (2010) 055029.

[10] G. Xianlong, R. Asgari, Phys. Rev. A 77 (2008) 033604.

[11] M. Rigol, A. Muramatsu, Phys. Rev. A 69 (2004) 053612.

[12] M. Casula, D. M. Ceperley, E. J. Mueller, Phys. Rev. A 78 (2008) 033607.

[13] X.Yin, Xi-Wen. Guan, Sh. Chen, M. T. Batchelor, Phys. Rev. A 84 (2011) 063632.

[14] M. Colome-Tatche, Phys. Rev. A 78 (2008) 033612.

[15] J. Reichel, J. H. J. Thywissen, Phys. IV. France. 116 (2004) 265-274.

[16] S. Hofferberth, I. Lesanovsky, B. Fischer, T. Schumm, J. Schmiedmayer, Nature. 449 (2007) 324-327.

[17] J. Fortagh, C. Zimmermann, Rev. Mod. Phys. 79 (2007) 235.

[18] Th. Stoferle, H. Moritz, Ch. Schori, M. Kohl, T. Esslinger, Phys. Rev. Lett. 92 (2004) 130403.

[19] S. Stock, Z. Hadzibabic, B. Battelier, M. Cheneau, J. Dalibard, Phys. Rev. Lett. 95 (2005) 190403.

[20] S. K. Baur, J. Shumway, E. J. Mueller, Phys. Rev. A 81 (2010) 3628.

[21] C. Chin, R. Grimm, P. S. Julienne, E. Tiesinga, Rev. Mod. Phys. 82 (2010) 1225.

[22] Y. Liao, A. C. Rittner, T. Li. W. Paprotta, G. B. Partridge, R. G. Hulet, S. K. Baur, E. J. Mueller, Nature. 467 (2010) 567-569.

[23] Y. Sagi, T. E. Drake, R. Paudel, R. Chapurin, and D. S. Jin. J. Phys: Conference Series 467 (2013) 012010 .

[24] Y. Lee, T. Wang, T. M. Rvachov, J. Choi, W. and M. Heo, Phys. Rev. A 87 (2013) 043629.

[25] A. L. Gaunt, T. F. Schmidutz, I. Gotlibovych, R. P. Smith, and Z. Hadzibabic, Phys. Rev. Lett. 110 (2013) 200406.

[26] I. Gotlibovych, T. F. Schmidutz, A. L. Gaunt, N. Navon, R. P. Smith, and Z. Hadzibabic, Phys. Rev. A 89 (2014) 061604 (R).

[27] A. Csordas, O. Almasy, and P. Szepfalusy, Phys. Rev. A 82 (2010) 063609.

[28] R. Haussmann and W. Zwerger, Phys. Rev. A 78 (2008) 063602.

[29] T. E. Drake, Y. Sagi, R. Paudel, J. T. Stewart, J. P. Gaebler, and D. S. Jin, Phys. Rev. A 86 (2012) 031601.

[30] Y. Sagi, T. E. Drake, R. Paudel, and D. S. Jin, Phys. Rev. Lett. 109 (2012) 220402.

[31] M. K. Al-Sugheir, Int. J. Theor. Phys. 43 (2004) 1527-1539.

[32] M. K. Al-Sugheir, H. B.Ghassib, Int. J. Theor. Phys. 41 (2002) 705719.

[33] M. K. Al-Sugheir, F. M. Al-Dweri, G. Alna'washi, M. G. Shatanawi, Physica B. 40 (2013) 151-157.

[34] M. K. Al-Sugheir, H. B. Ghassib, M. Awawdeh, Phys. Rev. A 84 (2011) 013617.

[35] B. R. Joudeh, M. K. Al-Sugheir, H. B. Ghassib, Int. J. Mod. Phys. B 19 (2005) 3985-4008.

[36] A. R. Sakhel, S.I. Qashou, R. R. Sakhel, H. B. Ghassib, Phys. Rev. A 82 (2010) 063618.

[37] A. R. Lima, (2010) Unpublished Doctoral Dissertation. Free University of Berlin, Germany.

[38] L. O. Baksmaty, H. Lu, C. J. Bolech, and H. Pu, New. J. Phys. 13 (2011) 055014.

[39] R. R. Nigmatullin, V. A. Toboev. Theor. Math. Phys. 80 (1989) 94106.

[40] R. R. Nigmatullin, A. A. Khamzin, H. B. Ghassib, Solid State Commun. 113 (2000a) 257-261.

[41] R. R. Nigmatullin, A. A. Khamzin, H. B. Ghassib, Int. J. Theor. Phys. 39 (2000b) 405-446.

[42] R. R. Nigmatullin, A. A. Khamzin, H. B. Ghassib, Phys. Rev. E 61 (2000c) 3441-3449.

[43] M. K. Al-Sugheir, A. S. Sandouqa, B. R. Joudeh, S. Al-Omari, M. Awawdeh, F. Rawwagah, Physica B. 405. (2010) 2171- 2174.

[44] M. K. Al-Sugheir, H. B. Ghassib, R. R Nigmatullin, Int. J. Theor. Phys. 40 (2001) 1033-1060.

[45] M. K. Al-Sugheir, G. Alna'washi, H. B. Ghassib, A. Sandouqa, Physica B. 407 (2012) 2313-2320.

[46] S. Giorgini, L. P. Pitaevskii, S. Stringari, Rev. Mod. Phys. 80 (2008) 1215.

[47] P. N. Vorontsov, R. I. Gorbunov, S. D. Ivanov, J. Phys. Math. and General. 33 (2000) 1857-1871.

[48] G. Su, J. Chen and L. Chen, Phys. Lett. A 315 (2003) 109-119.

[49] M. Grether, M. Fortes, M. de Llano, J. L. del Rio, F. J. Sevilla, M. A. Solis, A. A. Valladares, Eur. Phys. J. D 23 (2003) 117- 124.

[50] X.-J. Liu, H. Hu, P. D. Drummond, Phys. Rev. A 78 (2008) 023601.

[51] G. M. Bruun and K. Burnett, Phys. Rev . A 58 (1998) 2427-2434.

[52] D. A. Butts and D. S. Rokhsar, Phys. Rev. A 55 (1997) 05306-7. 
[53] M. Li, Z. Yan, J. Chen, L. Chen, Ch Chen, Phys. Rev. A 58 (1998) 1445-1449.

[54] A. Snyder, I. Tanabe, and Th. Silva, Phys. Rev. A 83 (2013) 063632.

[55] F. Qin and J. Chen, J. Phys. B 43 (2010) 055302.

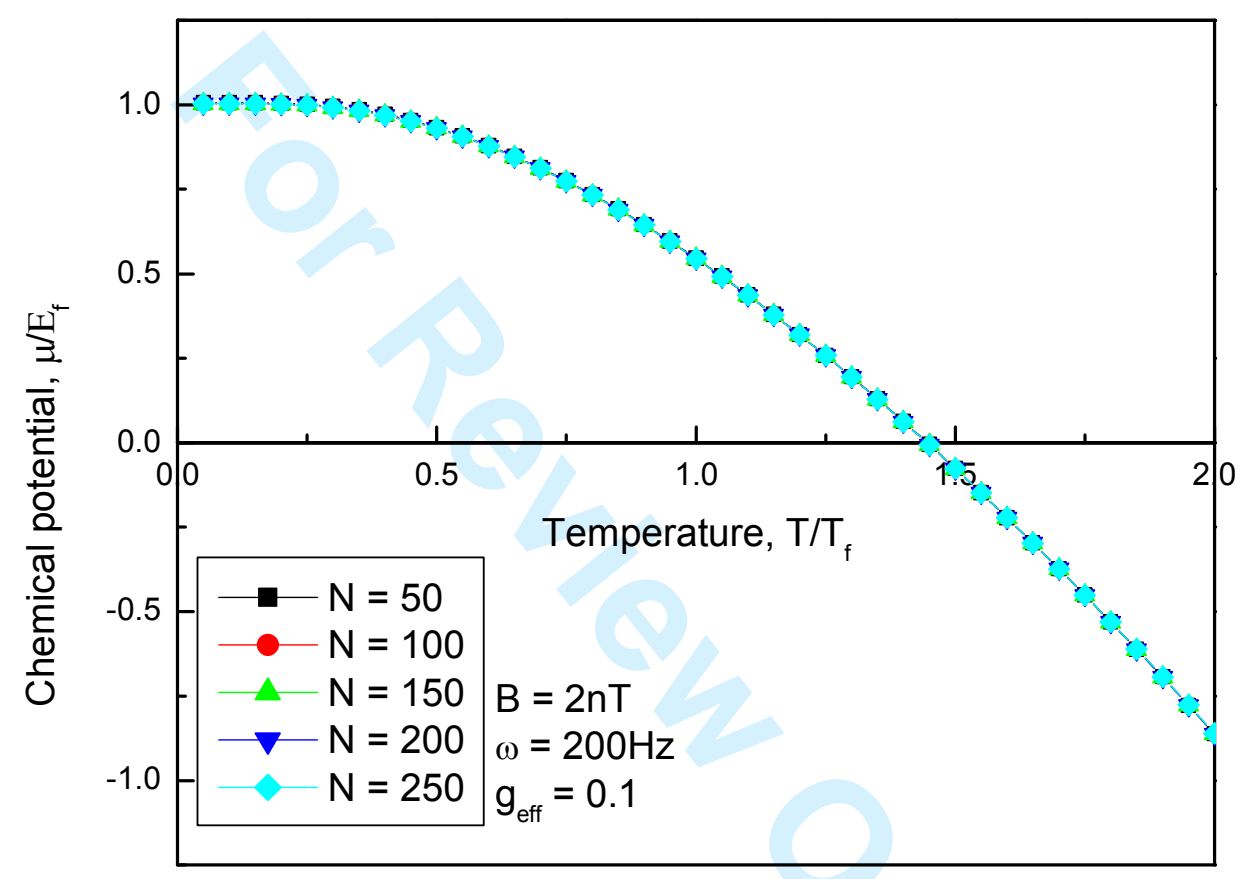

Fig. 1. The chemical potential $\mu / E_{F}$ as a function of temperature $T / T_{F}$ for different numbers of particles $N$. The frequency is $\omega=200 H z$, the magnetic field is $B=2 n T$ and the effective coupling constant is $g_{\text {eff }}=0.1$. 


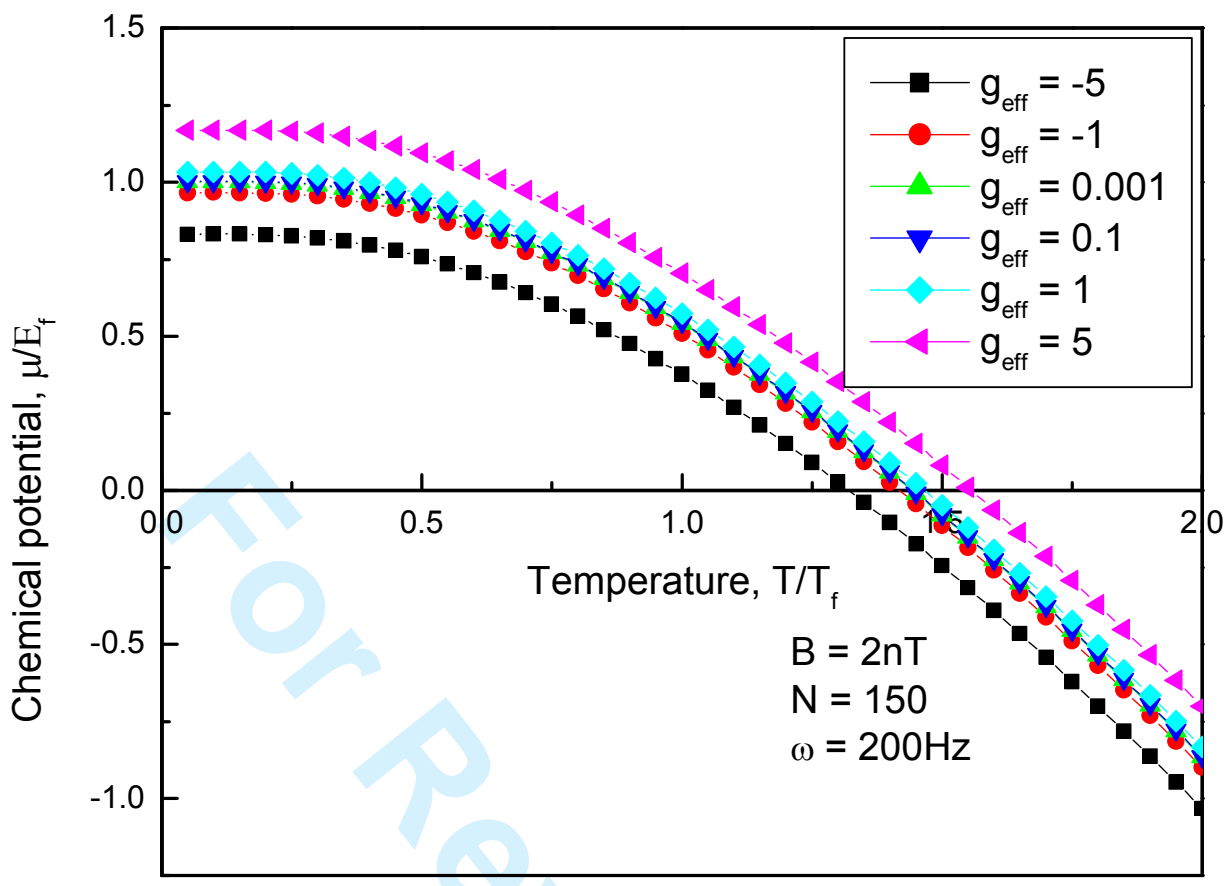

Fig. 2. The chemical potential $\mu / E_{F}$ as a function of temperature $T / T_{F}$ for different repulsive and attractive potential strengths $g_{\text {eff. }}$ The number of particles is $N=150$, the frequency is $\omega=200 \mathrm{~Hz}$ and the magnetic field is $B=2 n T$. 


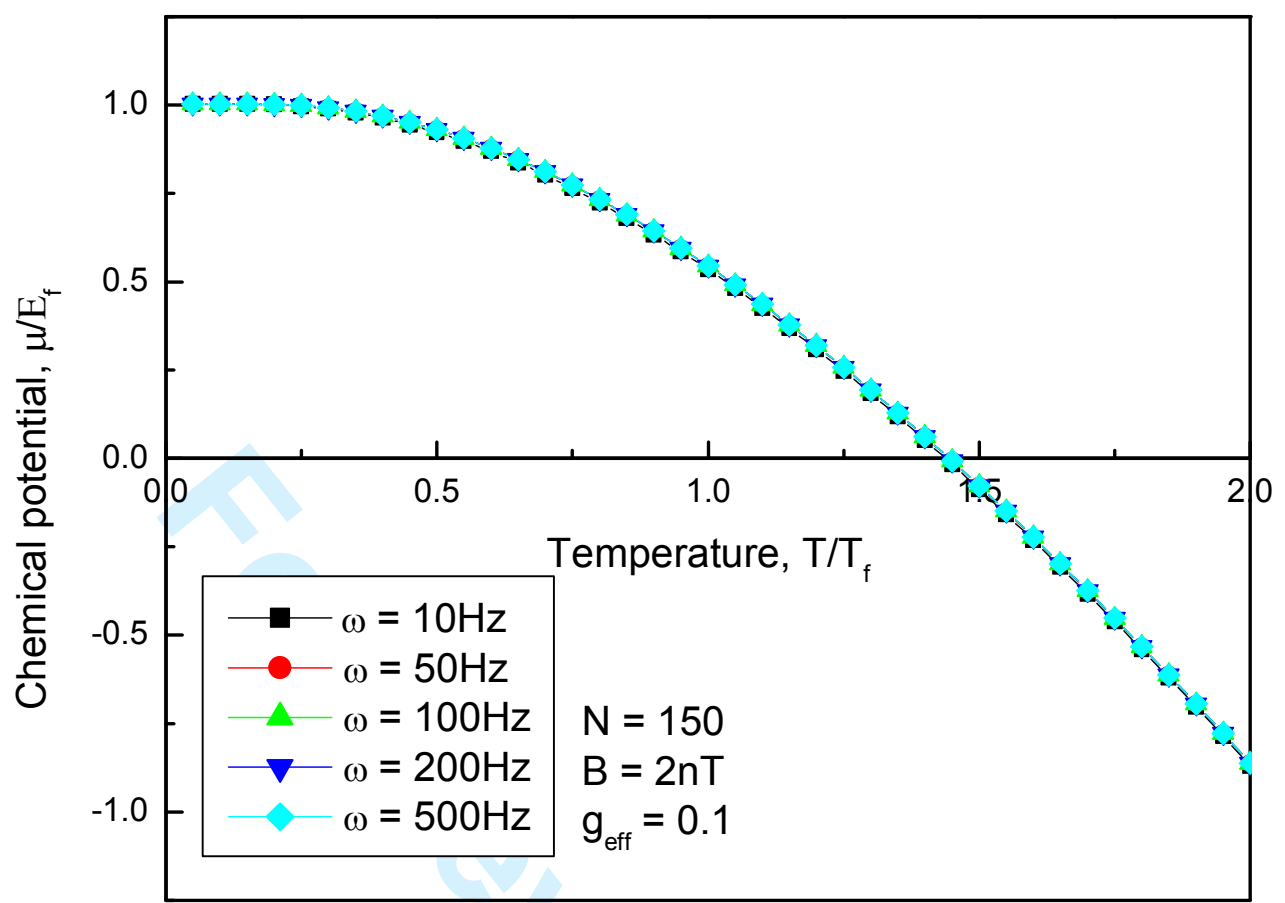

Fig. 3. The chemical potential $\mu / E_{F}$ as a function of temperature $T / T_{F}$ for different frequencies. The number of particles is $N=150$, the effective coupling constantis $g_{\text {eff }}=$ 0.1 and the magnetic field is $B=2 n T$. 


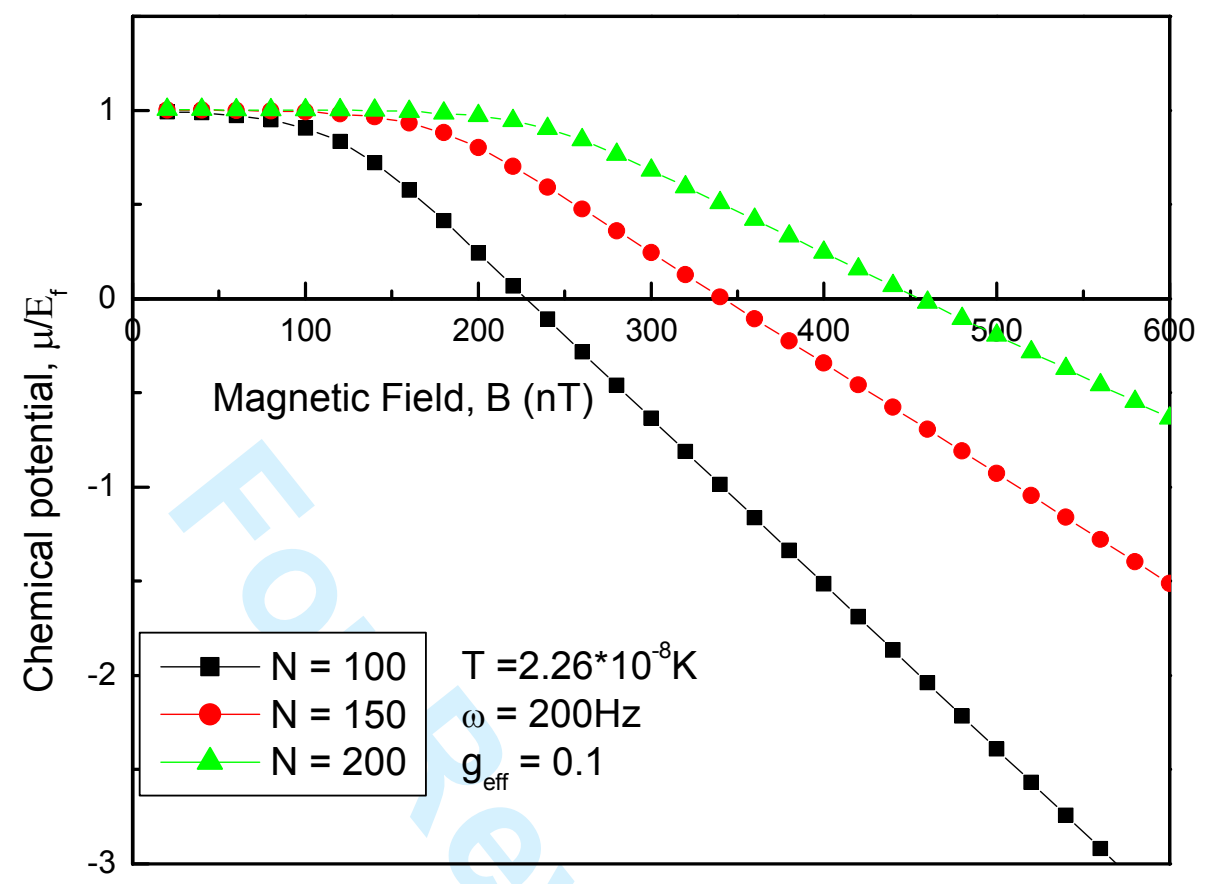

Fig. 4. The chemical potential $\mu / E_{F}$ as a function of magnetic field $B$ for different numbers of particles $N$. The frequency is $\omega=200 \mathrm{~Hz}$, the temperature is $T=22.6 \mathrm{nK}$ and the effective coupling constant is $g_{\text {eff }}=0.1$. 


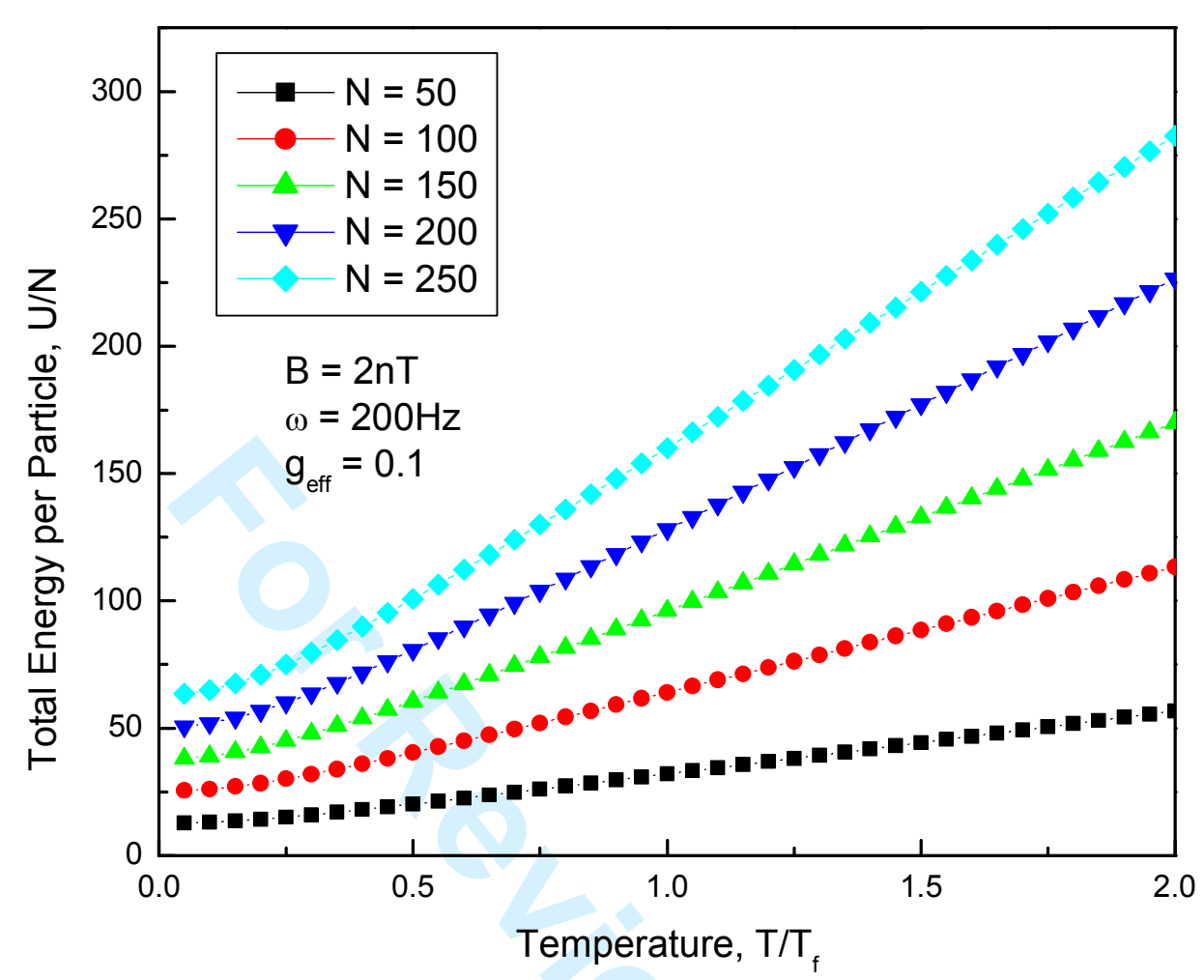

Fig. 5. The total energy per particle $U / N$ in trap units as a function of temperature $T / T_{F}$ for different numbers of particles $N$. The frequency is $\omega=200 \mathrm{~Hz}$, the magnetic field is $B=$ $2 n T$ and the effective coupling constant is $g_{\text {eff }}=0.1$. 


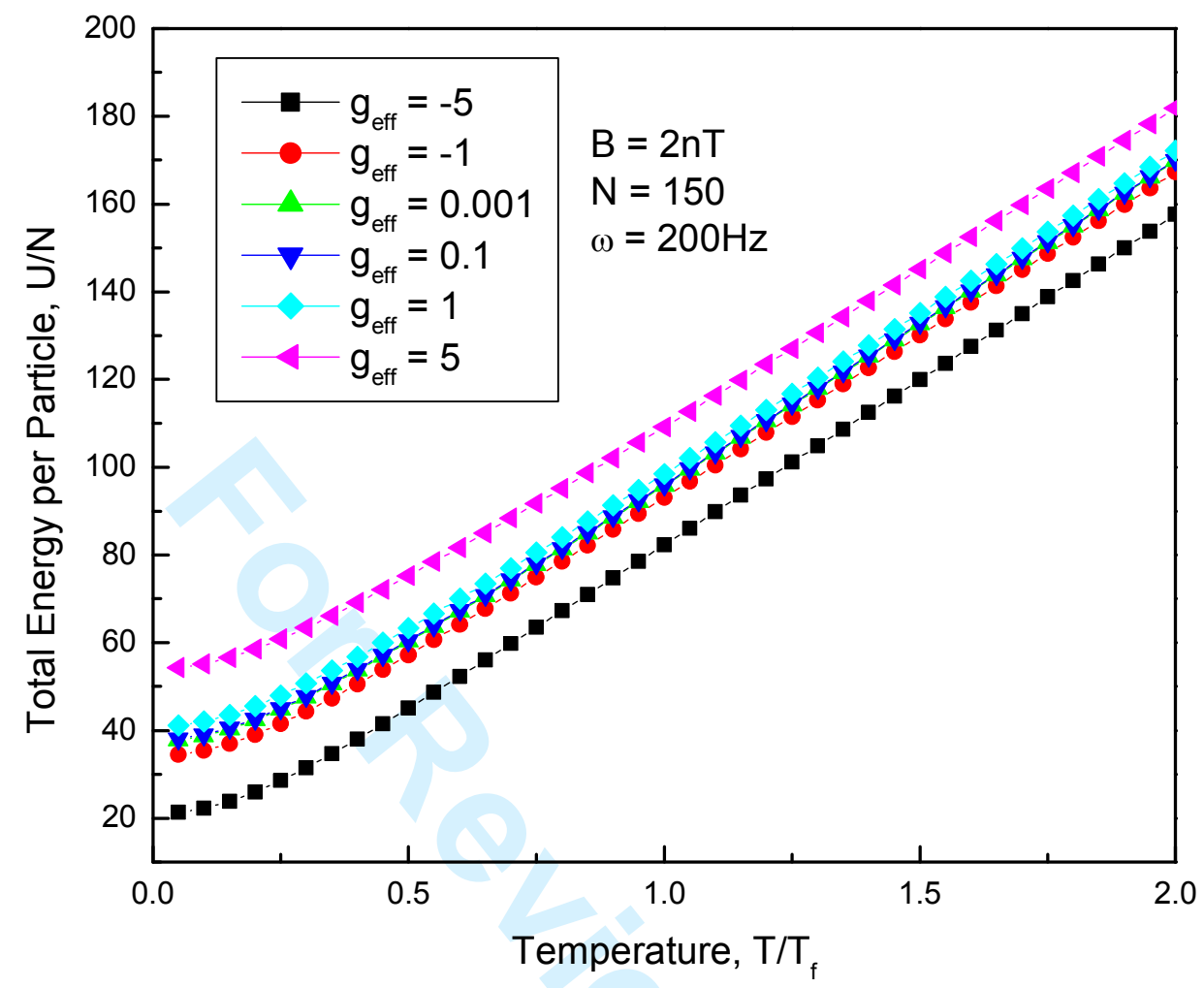

Fig. 6. The total energy per particle $U / N$ in trap units as a function of temperature $T / T_{F}$ for different repulsive and attractive potential strengths $g_{\text {eff. }}$. The number of particles is $N$ $=150$, the frequency is $\omega=200 \mathrm{~Hz}$ and the magnetic field is $B=2 n T$. 


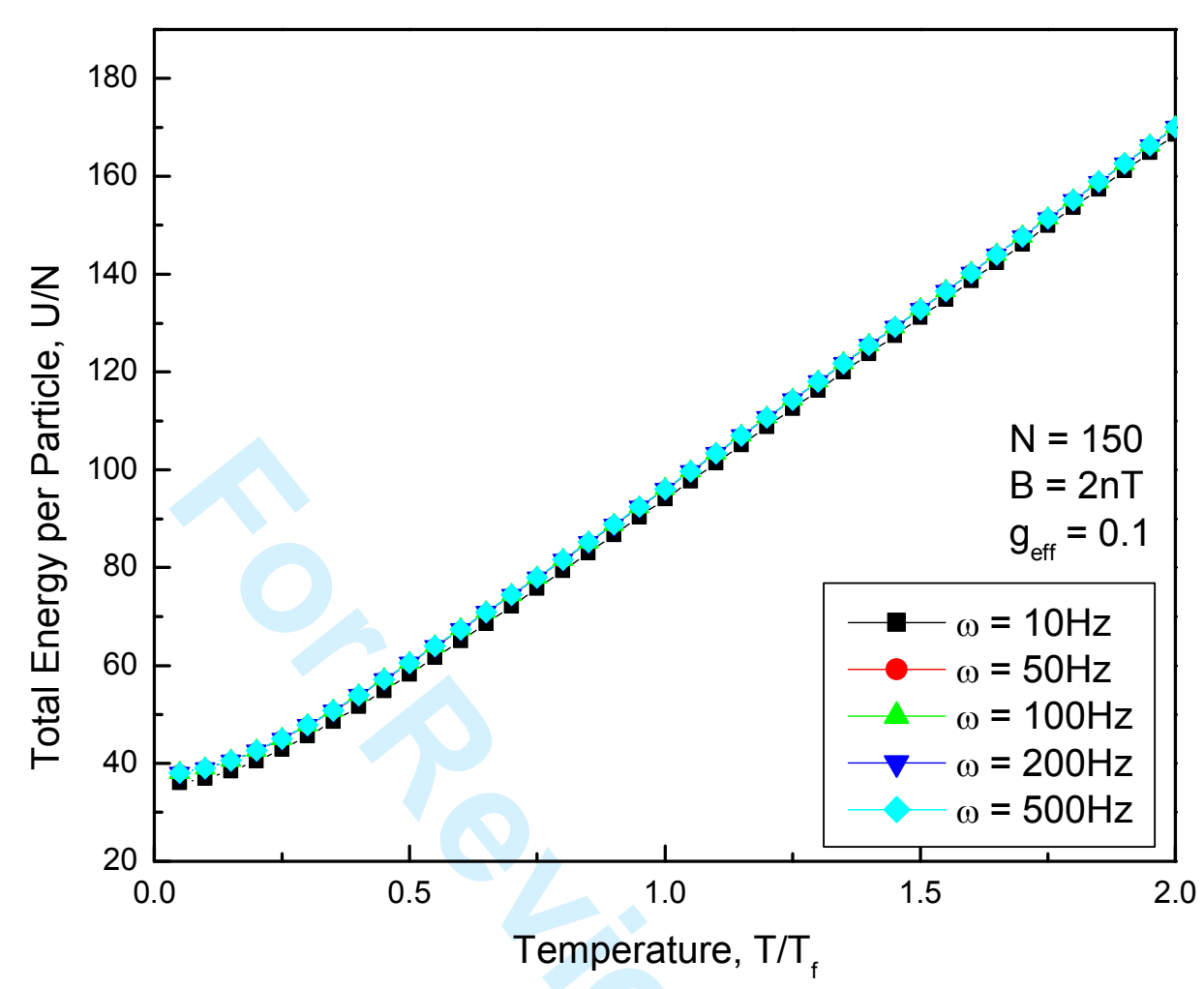

Fig. 7. The total energy per particle $U / N$ in trap units as a function of temperature $T / T_{F}$ for different frequencies $\omega$. The number of particles is $N=150$, the effective coupling constantis $g_{\text {eff }}=0.1$ and the magnetic field is $B=2 n T$. 


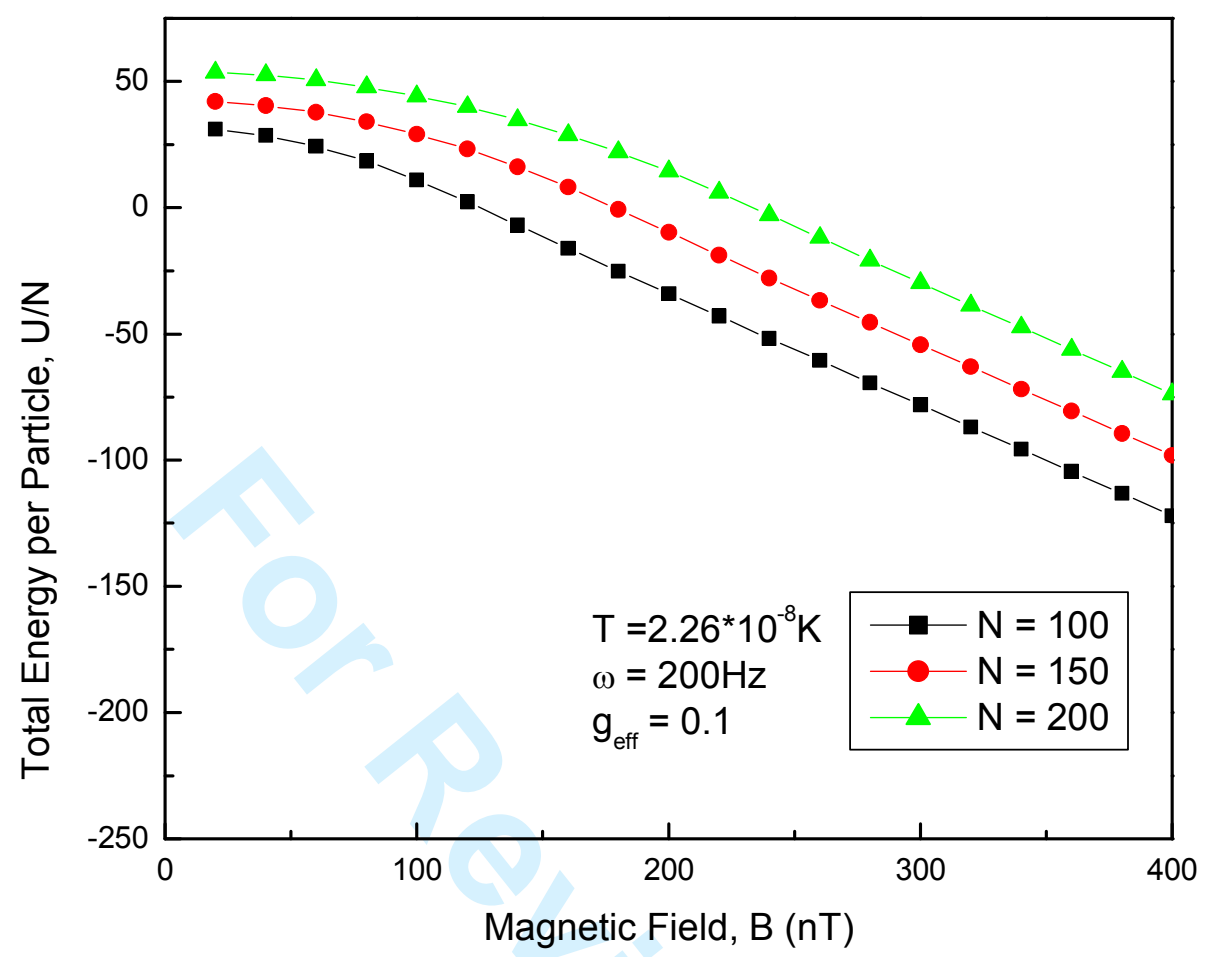

Fig. 8. The total energy per particle $U / N$ in trap units as a function of magnetic field $B$ for different numbers of particles $N$. The frequency is $\omega=200 \mathrm{~Hz}$, the temperature is $T=$ $22.6 n K$ and the effective coupling constant is $g_{\text {eff }}=0.1$. 


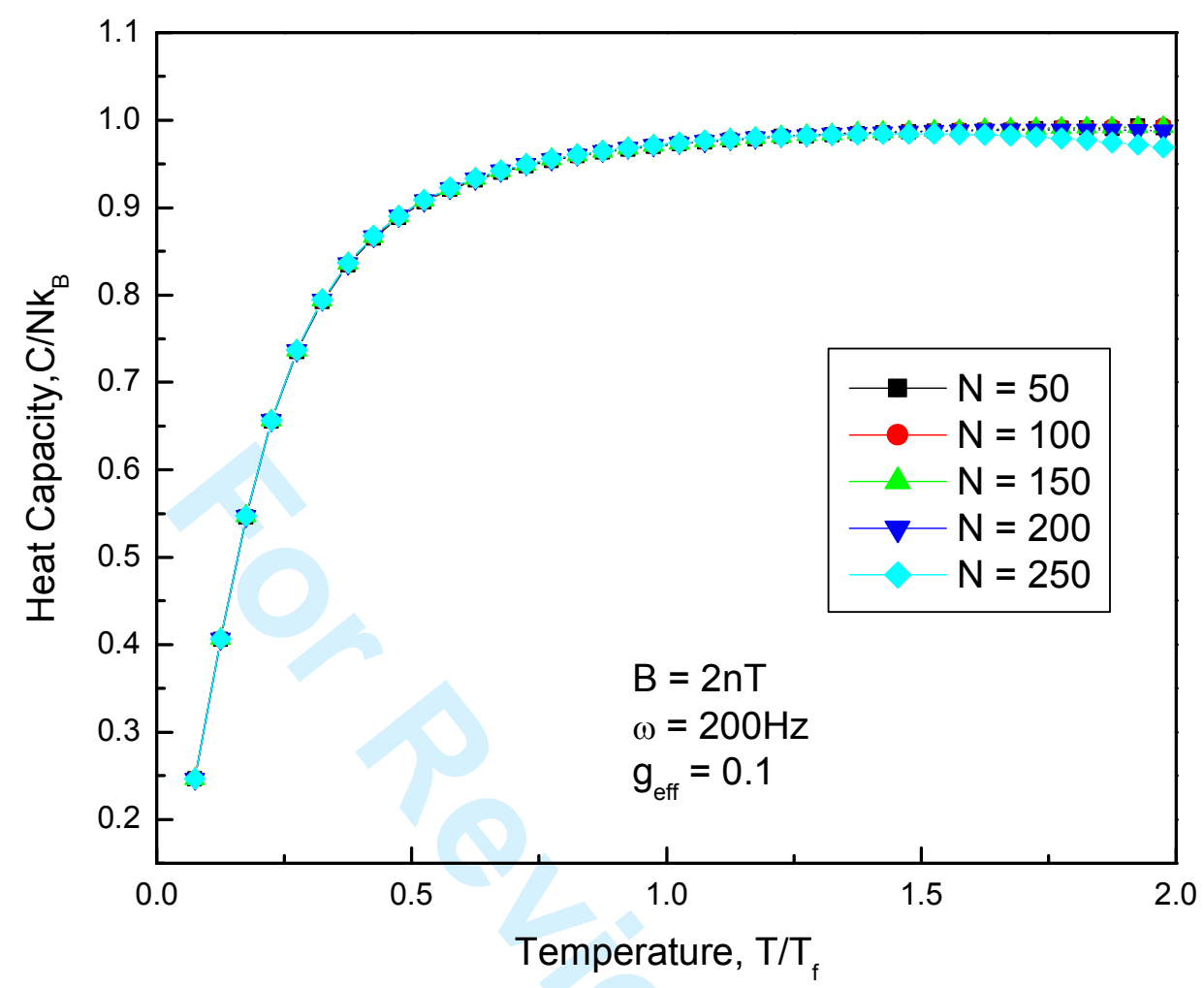

Fig. 9. The heat capacity $C / N k_{B}$ as a function of temperature $T / T_{F}$ for different numbers of particles $N$. The frequency is $\omega=200 \mathrm{~Hz}$, the magnetic field is $B=2 n T$ and the effective coupling constant is $g_{\text {eff }}=0.1$. 


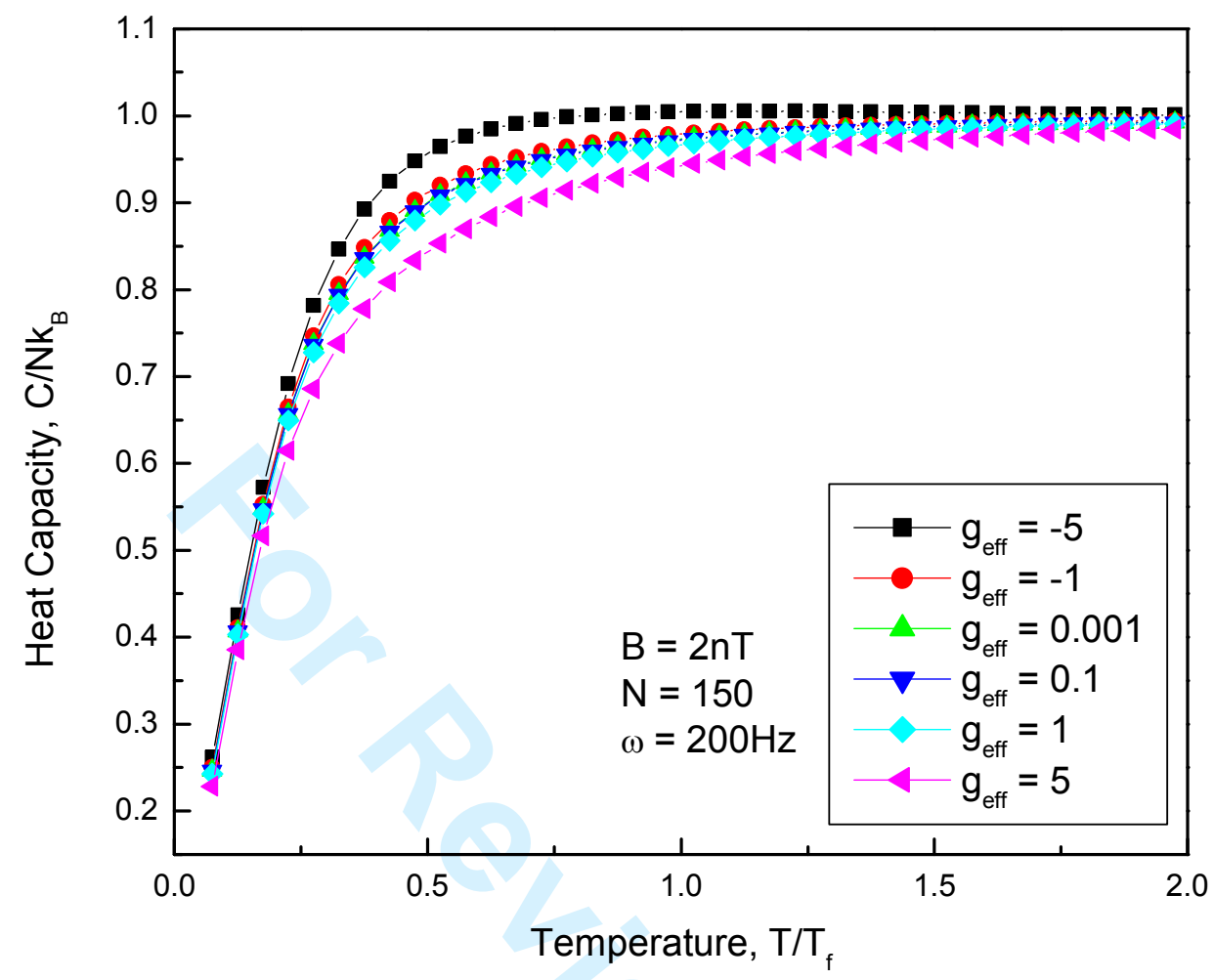

Fig. 10. The heat capacity $C / N k_{B}$ as a function of temperature $T / T_{F}$ for different repulsive and attractive potential strengths $g_{\text {eff. }}$. The number of particles is $N=150$, the frequency is $\omega=200 \mathrm{~Hz}$ and the magnetic field is $B=2 n T$. 


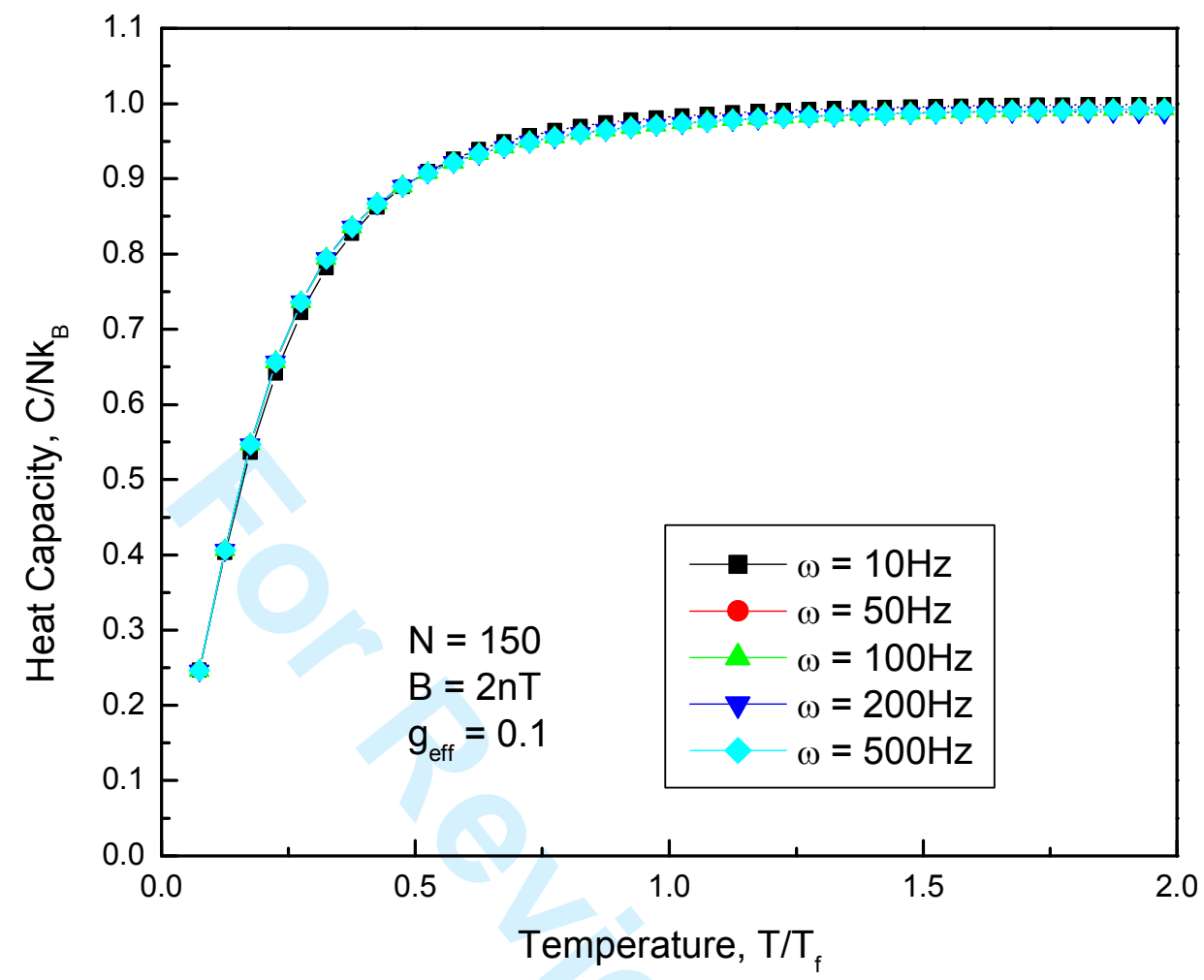

Fig. 11. The heat capacity $C / N k_{B}$ as a function of temperature $T / T_{F}$ for different frequencies $\omega$. The number of particles is $N=150$, the effective coupling constantis $g_{\text {eff }}=$ 0.1 and the magnetic field is $B=2 n T$. 


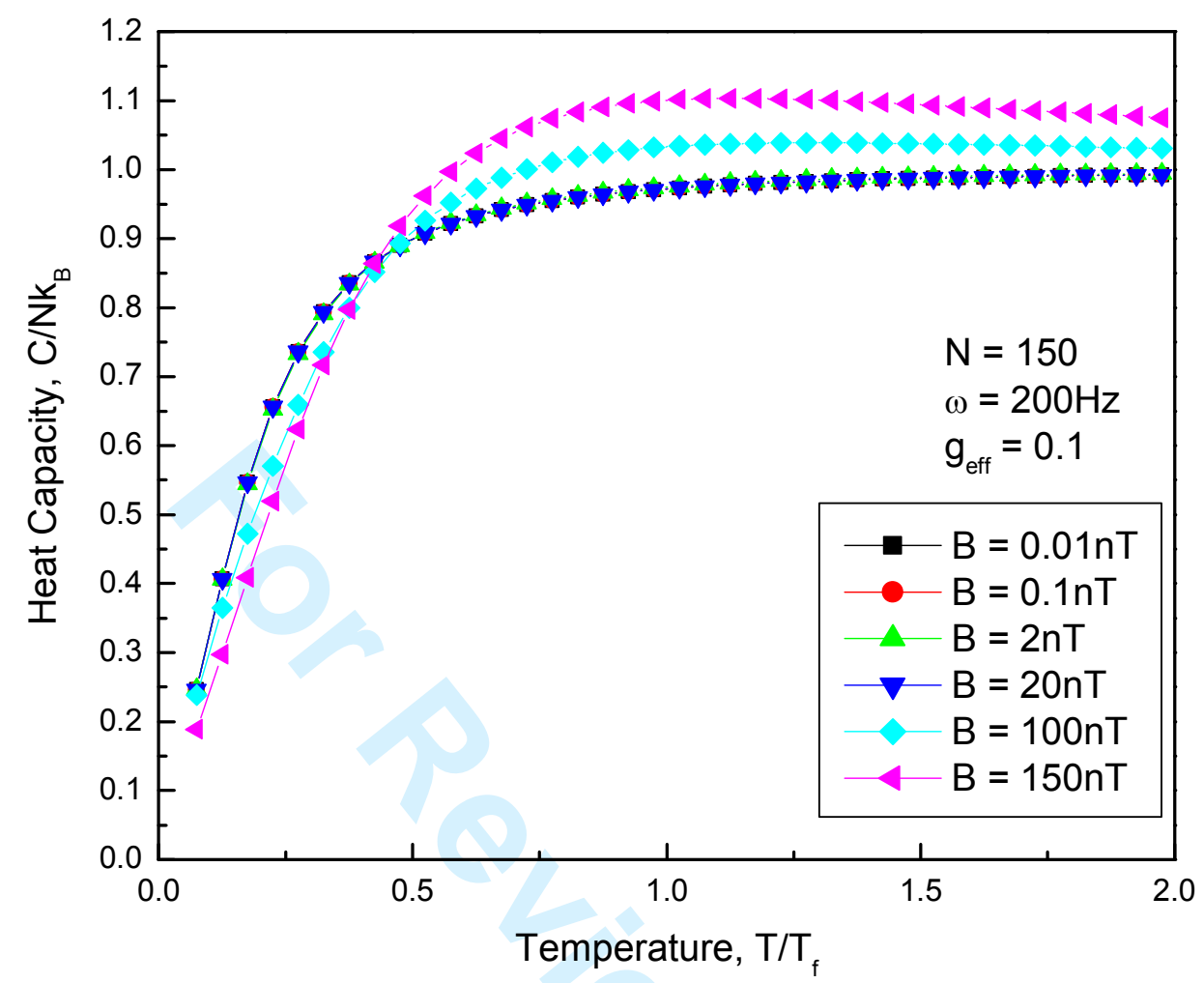

Fig. 12. The heat capacity $C / N k_{B}$ as a function of temperature $T / T_{F}$ for different magnetic fields $B$. The number of particles is $N=150$, the frequency is $\omega=200 \mathrm{~Hz}$ and the effective coupling constant is $g_{\text {eff }}=0.1$. 


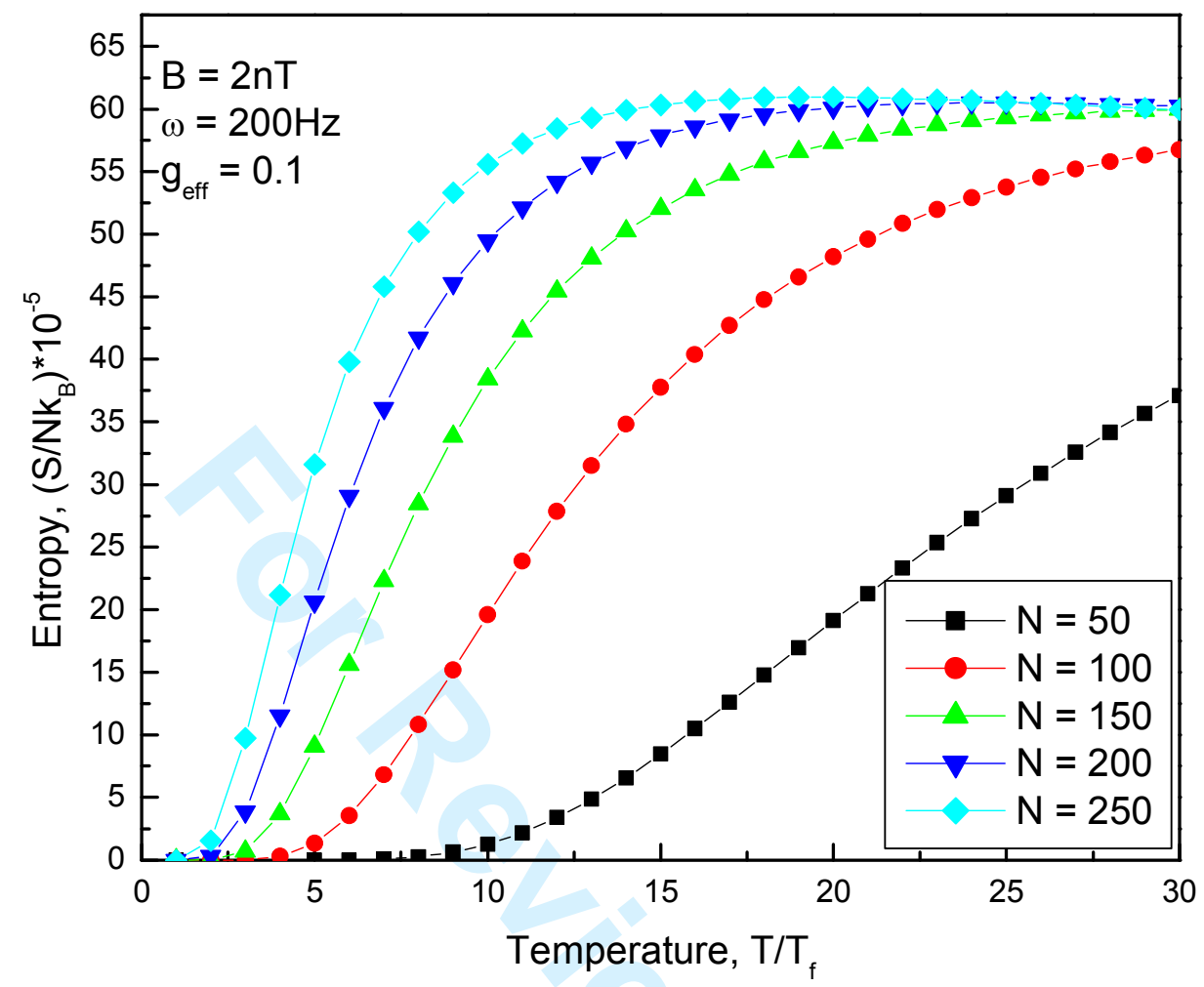

Fig. 13. The entropy $S / N k_{B}$ as a function of temperature $T / T_{F}$ for different numbers of particles $N$. The frequency is $\omega=200 H z$, the magnetic field is $B=2 n T$ and the effective coupling constant is $g_{\text {eff }}=0.1$. 


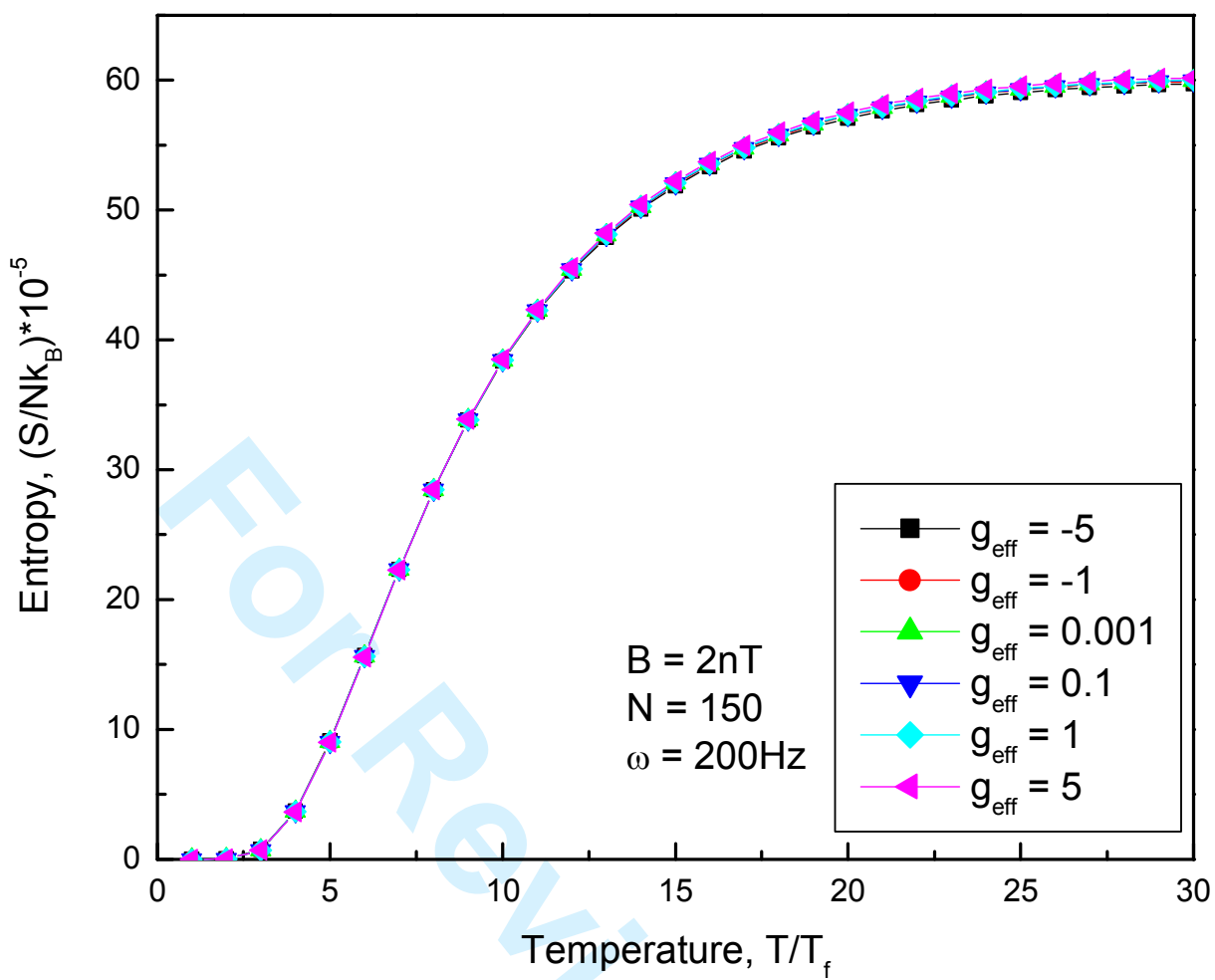

Fig. 14. The entropy $S / N k_{B}$ as a function of temperature $T / T_{F}$ for different repulsive and attractive potential strengths $g_{\text {eff. }}$ The number of particles is $N=150$, the frequency is $\omega=$ $200 \mathrm{~Hz}$ and the magnetic field is $B=2 n T$. 


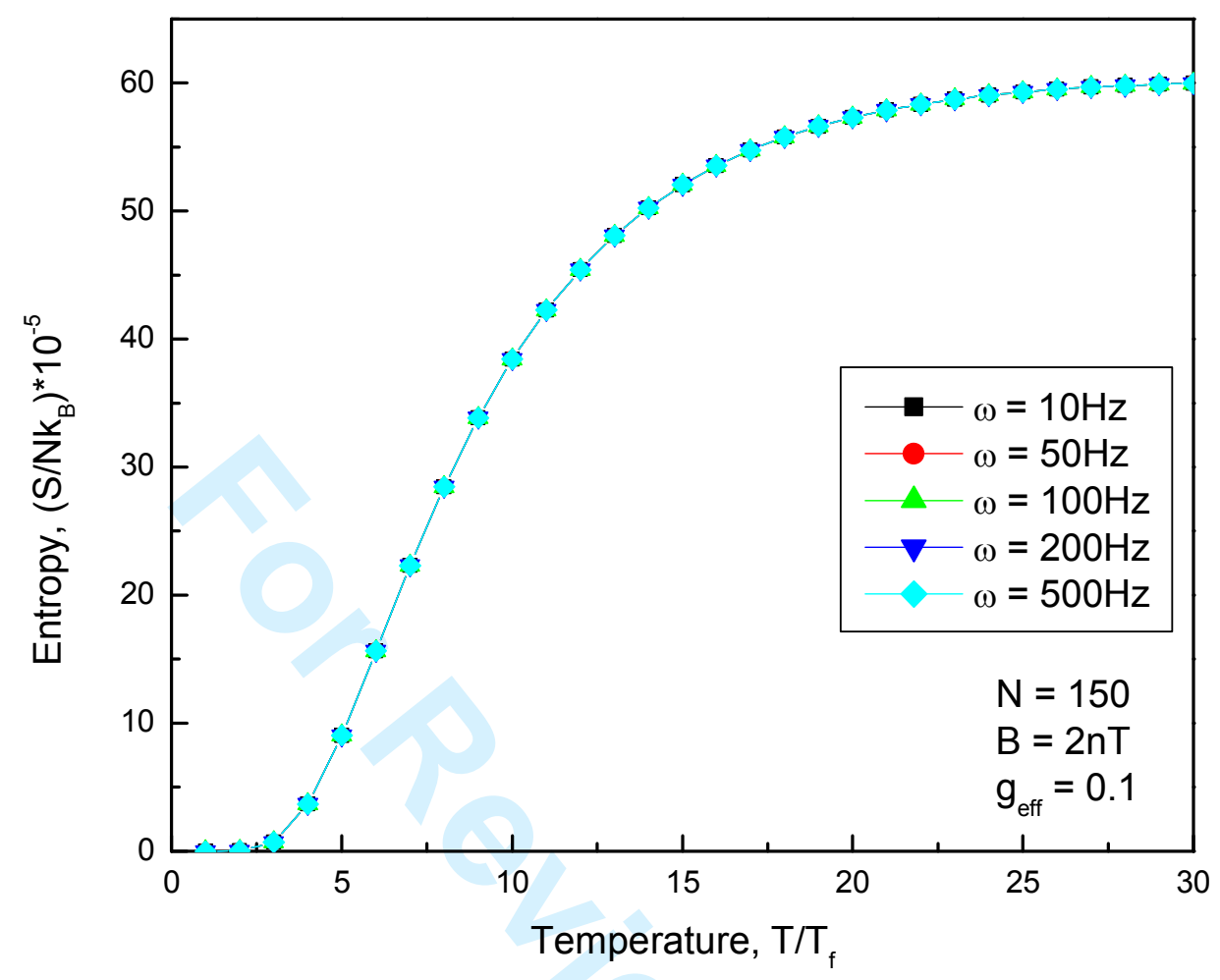

Fig. 15. The entropy $S / N k_{B}$ as a function of temperature $T / T_{F}$ for different frequencies $\omega$. The number of particles is $N=150$, the effective coupling constant is $g_{\text {eff }}=0.1$ and the magnetic field is $B=2 n T$. 


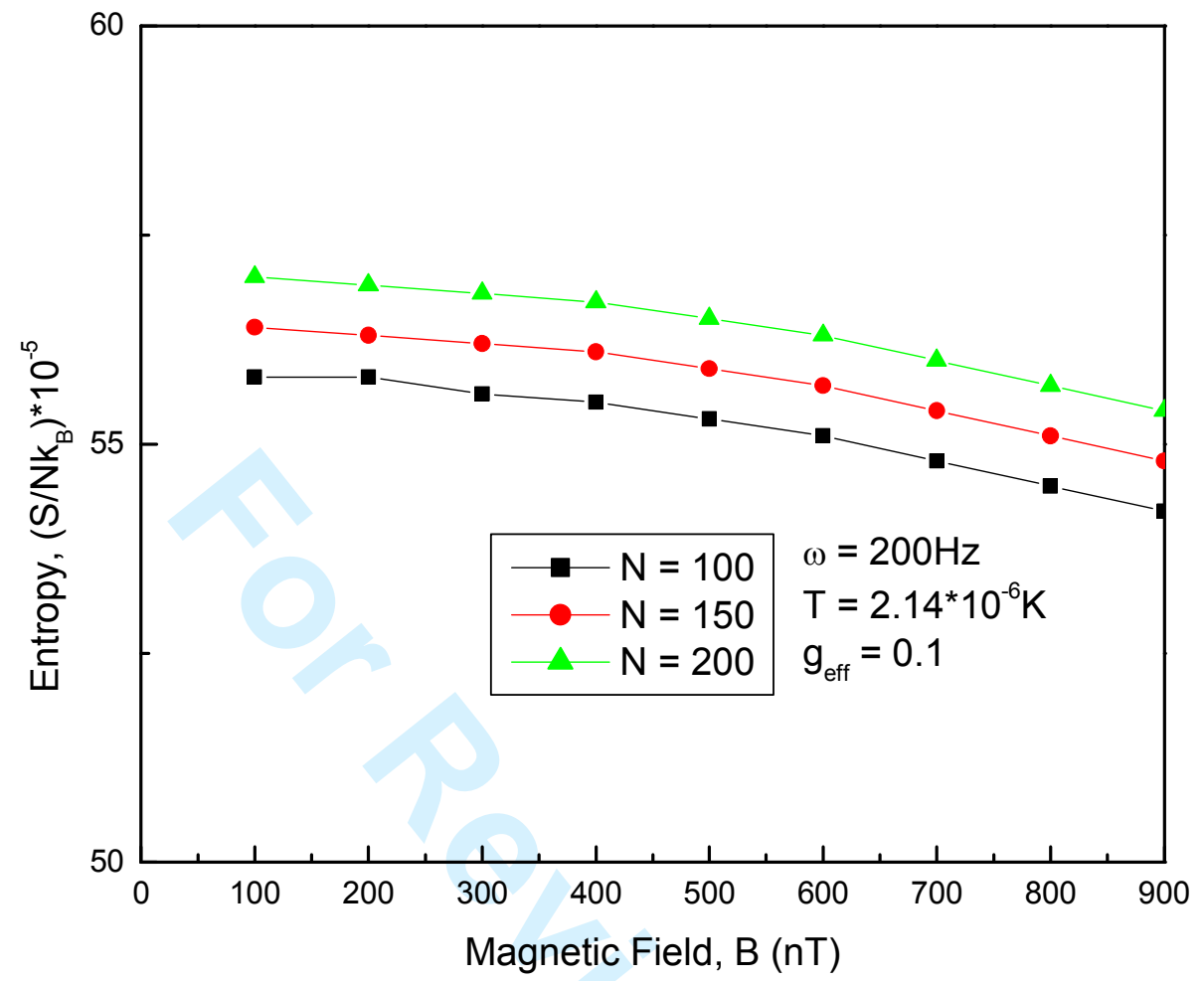

Fig. 16. The entropy $S / N k_{B}$ as a function of magnetic field $B$ for different numbers of particles $N$. The frequency is $\omega=200 \mathrm{~Hz}$, the temperature is $T=2.14 \mu \mathrm{K}$ and the effective coupling constant is $g_{\text {eff }}=0.1$. 


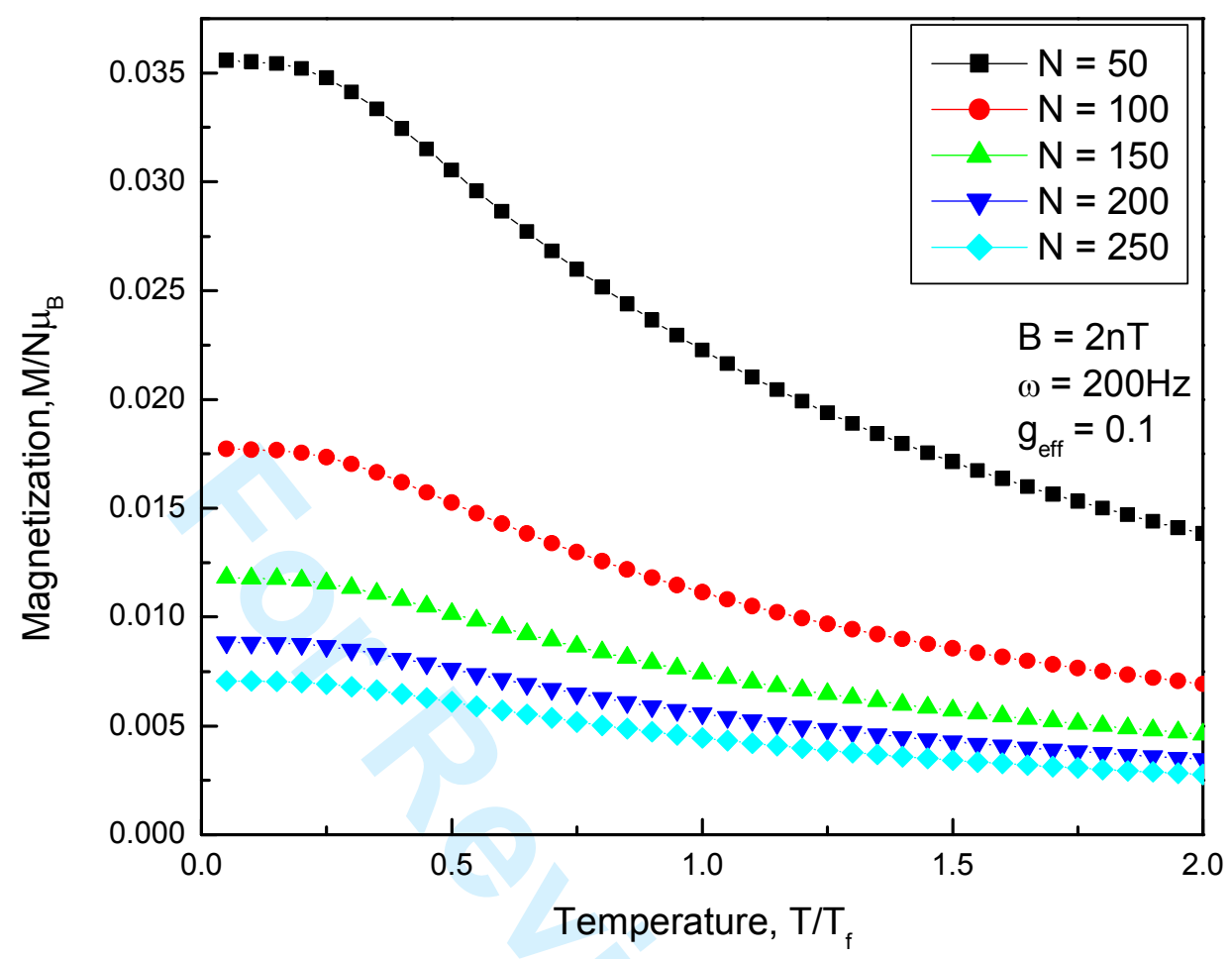

Fig. 17. The magnetization $M / N \mu_{B}$ as a function of temperature $T / T_{f}$ for different numbers of particles $N$. The frequency is $\omega=200 \mathrm{~Hz}$, the magnetic field is $B=2 n T$ and the effective coupling constant is $g_{\text {eff }}=0.1$. 


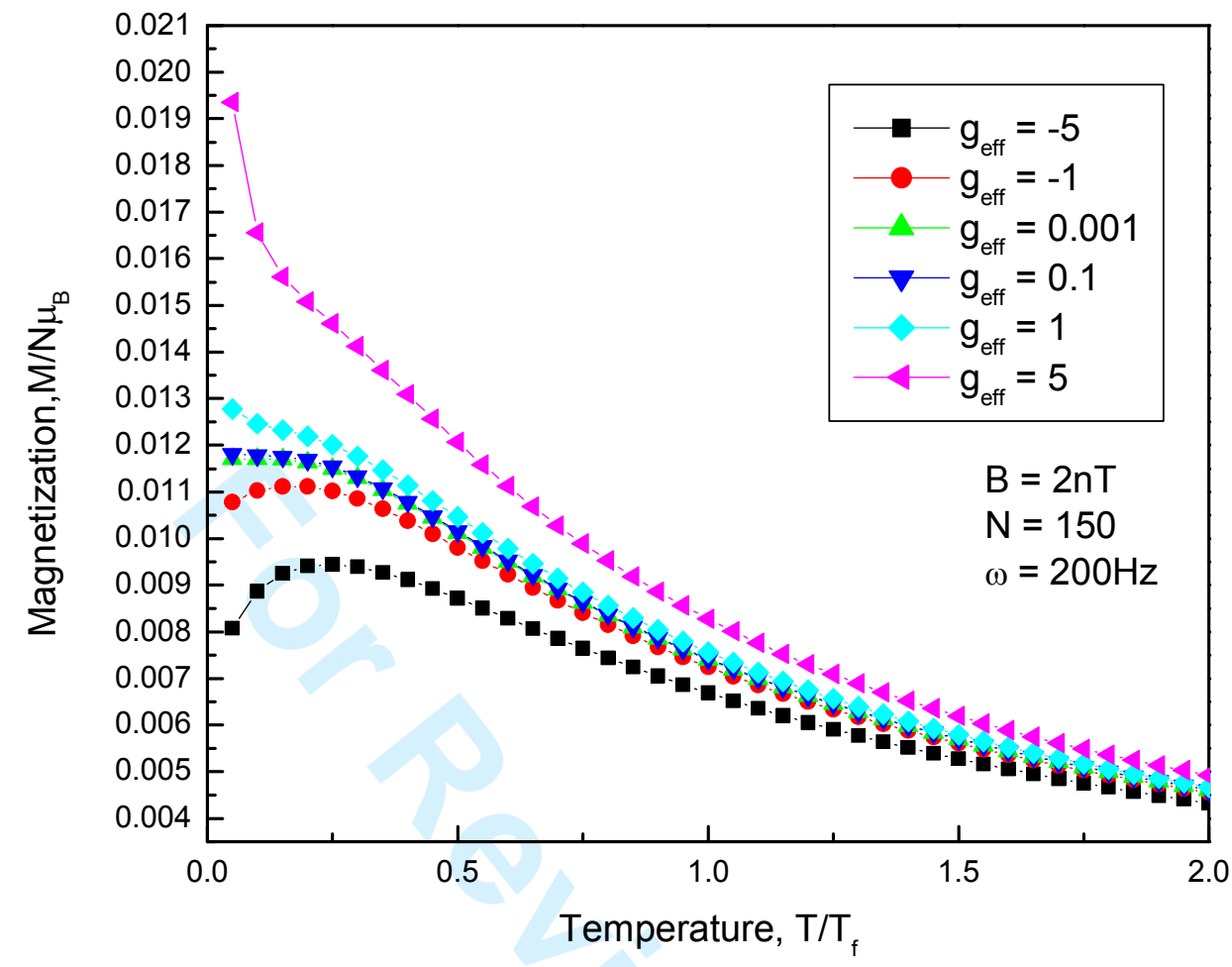

Fig. 18. The magnetization $M / N \mu_{B}$ as a function of temperature $T / T_{f}$ for different repulsive and attractive potential strengths $g_{\text {eff. }}$ The number of particles is $N=150$, the frequency is $\omega=200 \mathrm{~Hz}$ and the magnetic field is $B=2 n T$. 


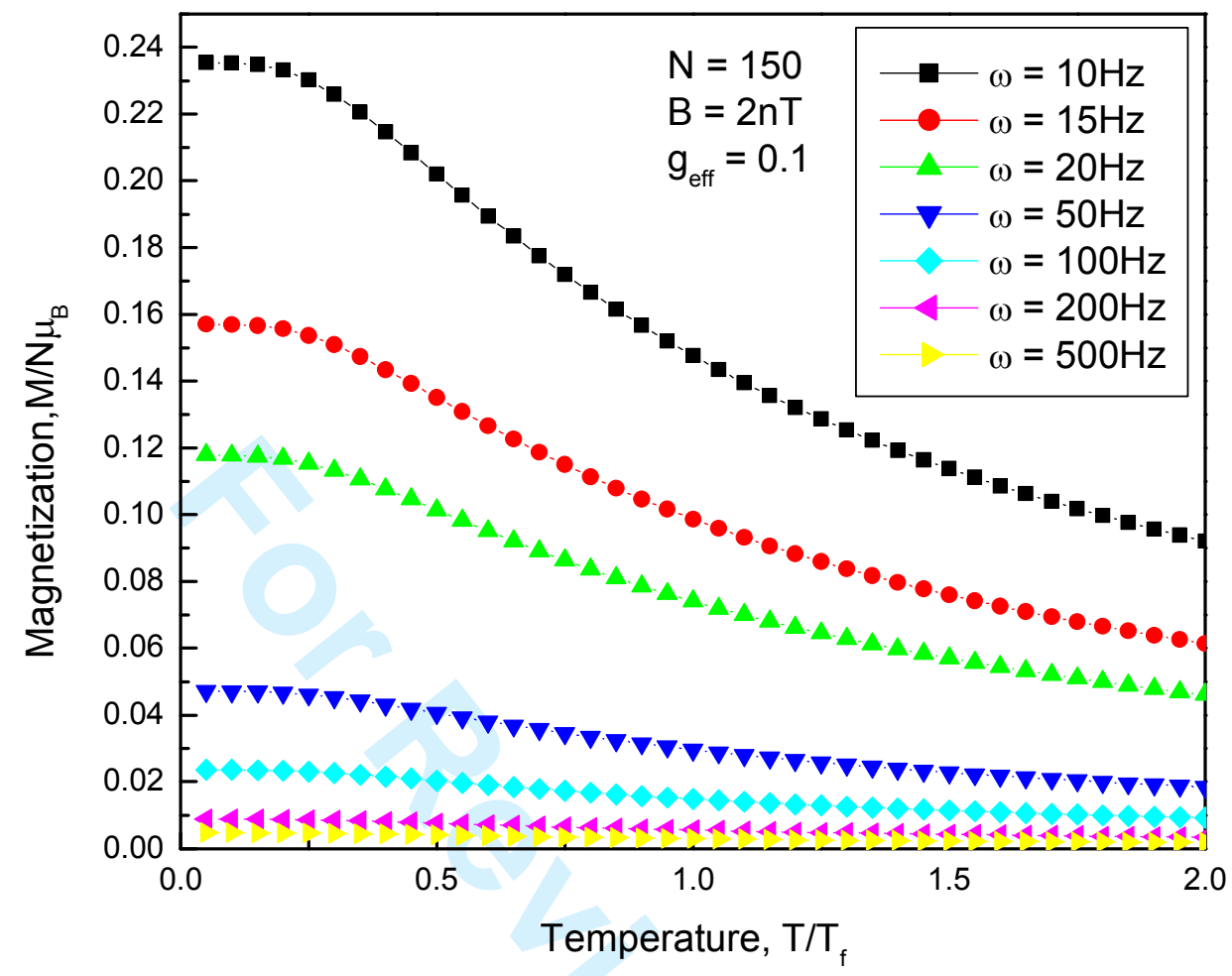

Fig. 19. The magnetization $M / N \mu_{B}$ as a function of temperature $T / T_{F}$ for different frequencies $\omega$. The number of particles is $N=150$, the effective coupling constantis $g_{\text {eff }}=$ 0.1 and the magnetic field is $B=2 n T$. 


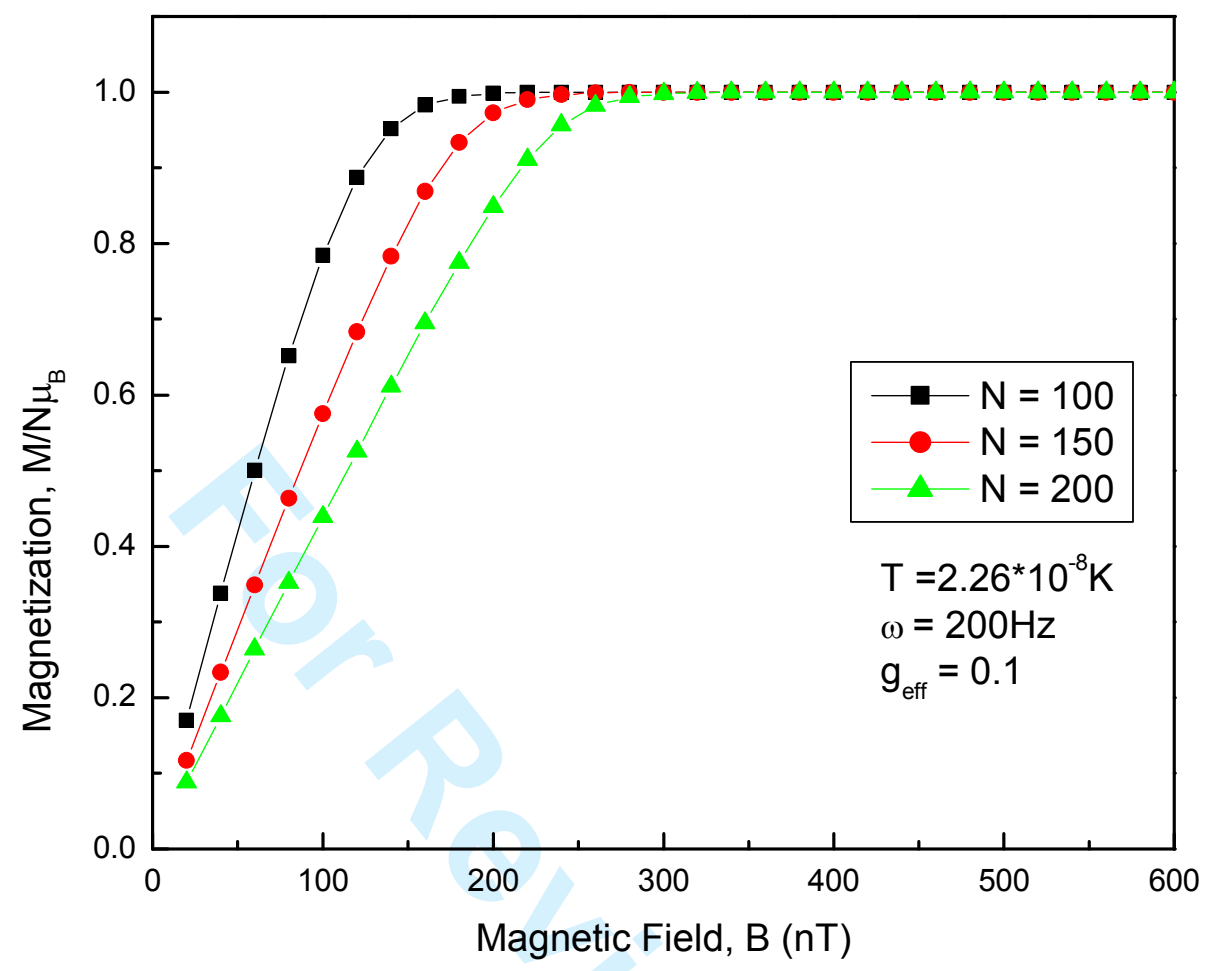

Fig. 20. The magnetization $M / N \mu_{B}$ as a function of magnetic field $B$ for different numbers of particles $N$. The frequency is $\omega=200 \mathrm{~Hz}$, the temperature is $T=22.6 n \mathrm{~K}$ and the effective coupling constant is $g_{\text {eff }}=0.1$. 


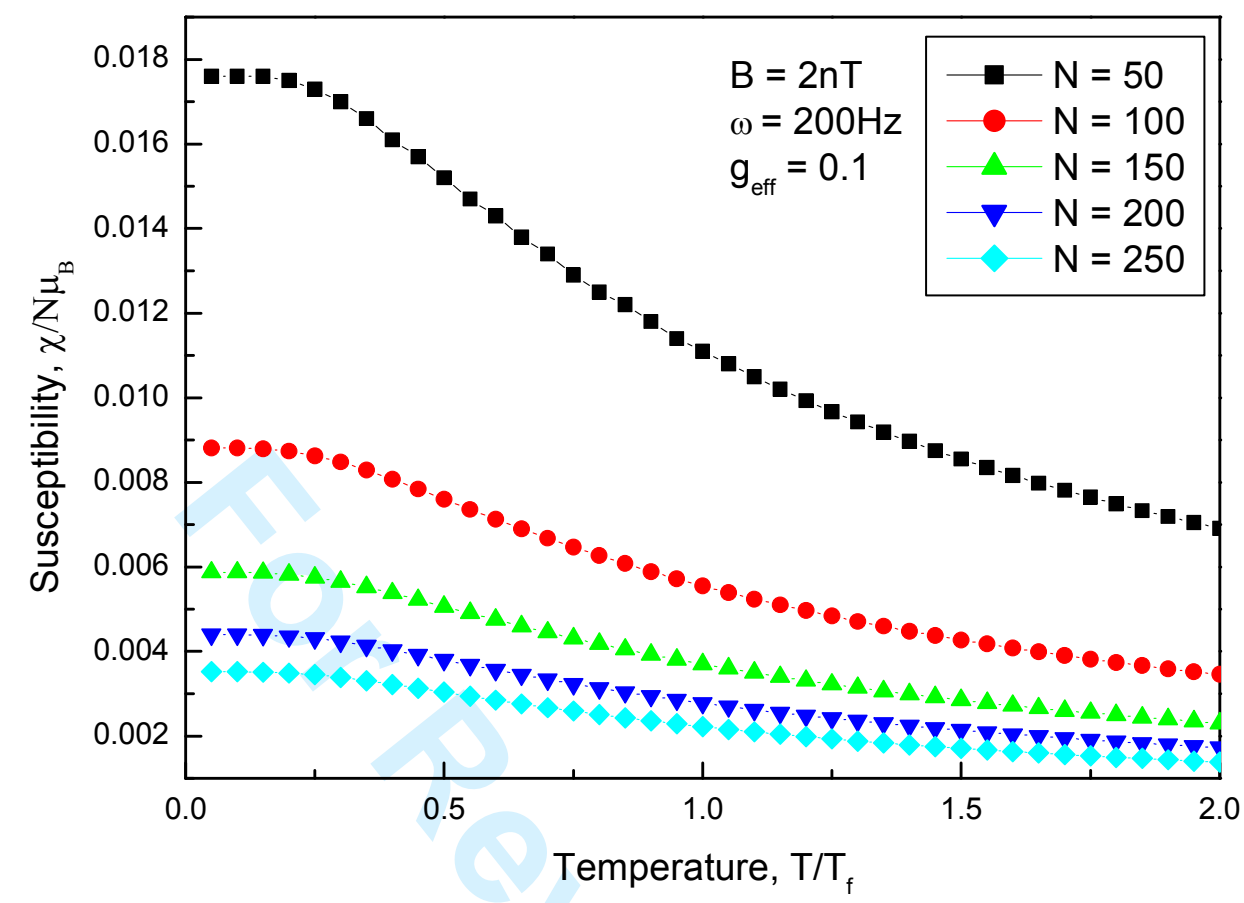

Fig. 21. The susceptibility $\chi / N \mu_{B}$ in units of $(1 / n T)$ as a function of temperature $T / T_{F}$ for different numbers of particles $N$. The frequency is $\omega=200 \mathrm{~Hz}$, the magnetic field is $B=$ $2 n T$ and the effective coupling constant is $g_{\text {eff }}=0.1$. 